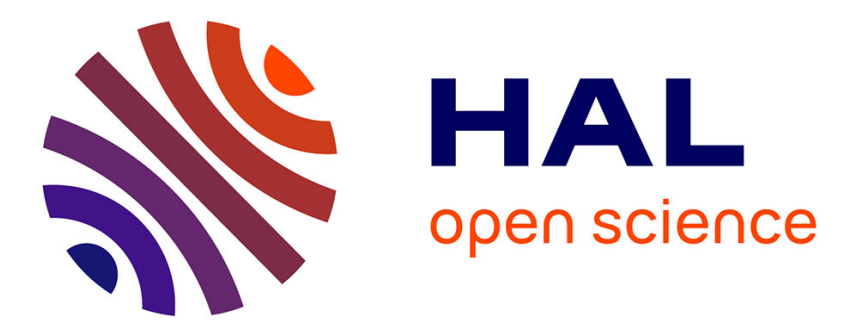

\title{
Automatic determination of the electron density measured by the relaxation sounder on board ISEE 1
}

\author{
Jean-Gabriel Trotignon, J. Etcheto, J.P. Thouvenin
}

\section{To cite this version:}

Jean-Gabriel Trotignon, J. Etcheto, J.P. Thouvenin. Automatic determination of the electron density measured by the relaxation sounder on board ISEE 1. Journal of Geophysical Research Space Physics, 1986, 91 (A4), pp.4302. 10.1029/JA091iA04p04302 . insu-02994631

\section{HAL Id: insu-02994631 \\ https://hal-insu.archives-ouvertes.fr/insu-02994631}

Submitted on 8 Nov 2020

HAL is a multi-disciplinary open access archive for the deposit and dissemination of scientific research documents, whether they are published or not. The documents may come from teaching and research institutions in France or abroad, or from public or private research centers.
L'archive ouverte pluridisciplinaire $\mathbf{H A L}$, est destinée au dépôt et à la diffusion de documents scientifiques de niveau recherche, publiés ou non, émanant des établissements d'enseignement et de recherche français ou étrangers, des laboratoires publics ou privés. 


\title{
Automatic Determination of the Electron Density Measured by the Relaxation Sounder on Board ISEE 1
}

\author{
J. G. Trotignon
}

Laboratoire de Physique et Chimie de l'Environnement, Orléans, France

\section{J. ЕTCHETO}

Centre de Recherche en Physique de l'Environnement Terrestre et Planétaire, Issy les Moulineaux, France

\section{J. P. Thouvenin}

Centre National d'Etudes Spatiales, Toulouse, France

\begin{abstract}
Relaxation sounders have proved to be successful in measuring the electron density in dilute space plasmas. The resonances excited at the characteristic frequencies of the medium depend very much on the local plasma. We present here methods to determine automatically, by a computer, the plasma frequency in the various regions encountered. In the solar wind and in the magnetosheath, only one strong, long-lasting resonance appears close to the plasma frequency $f_{\text {pe }}$ yielding directly the electron density. We use a pattern recognition method based on Sebestyen's discrimination approach, and each identified resonance is given with a quality criterion. From one year of data there is no drift of the resonance features, and the method is used with a success rate of $95 \%$ as determined by manual means. In the magnetosphere various types of resonances are excited at the characteristic frequencies of the medium (cyclotron harmonic (requencies $n f_{c e}$ and maximum frequencies of the Bernstein's modes $f_{a \text { ) }}$. We compare the dispersion relation of electrostatic waves, which contains $f_{c e}$ and $f_{p e}$ as parameters, to the observed frequencies of the resonances. First $f_{c e}$ is extracted using the harmonicity of the $n f_{c e}$ resonances, and then the $f_{\text {n }}$ series is compared to the dispersion relation for various values of $f_{\text {en }}$. In $93 \%$ of 250 cases the error for $f_{p e}$ is less than $10 \%$. In the magnetotail where the resonances are closely spaced in frequency, the frequency resolution of the experiment does not allow us to use this method and we determine $f_{\text {pe }}$ by the enhancement of the resonances power.
\end{abstract}

\section{INTRODUCTION}

During the last twenty years the relaxation sounder was undoubtedly a part of the most used active experiments for the study of spatial plasmas. After proving to be successful in ionospheric dense plasma (see Calvert [1969], Muldrew [1972a,b], McAfee [1973], and Benson [1977] for reviews), it was taken aboard spacecraft flown in the dilute plasma of the outer magnetosphere: GEOS 1 (launched in May 1977), ISEE 1 (October 1977) and GEOS 2 (July 1978). Here again, the results obtained were of excellent quality [Etcheto and Petit, 1977; Etcheto and Bloch, 1978]. Finally it was on board ISEE 1 that such an experiment was used for the first time outside the magnetosphere and proved to work very well in the solar wind and magnetosheath [Harvey et al., 1979].

The sounder transmits on a swept frequency and receives echoes after a measured delay in a way analogous to groundbased ionosondes. The transmitter excites natural modes of oscillation in the surrounding medium. Hence with suitable interpretation, we have a powerful tool for spatial plasma diagnostic.

The aim of the present paper is to handle several years of ISEE 1 data using a computer and to determine automatically a fundamental parameter, the plasma frequency, from the raw data. This data processing was essential to change the relaxation sounder into an instrument usable for routinely measuring the total electron density on board a spacecraft.
PRINCIPLE OF THE RELAXATION SOUNDER

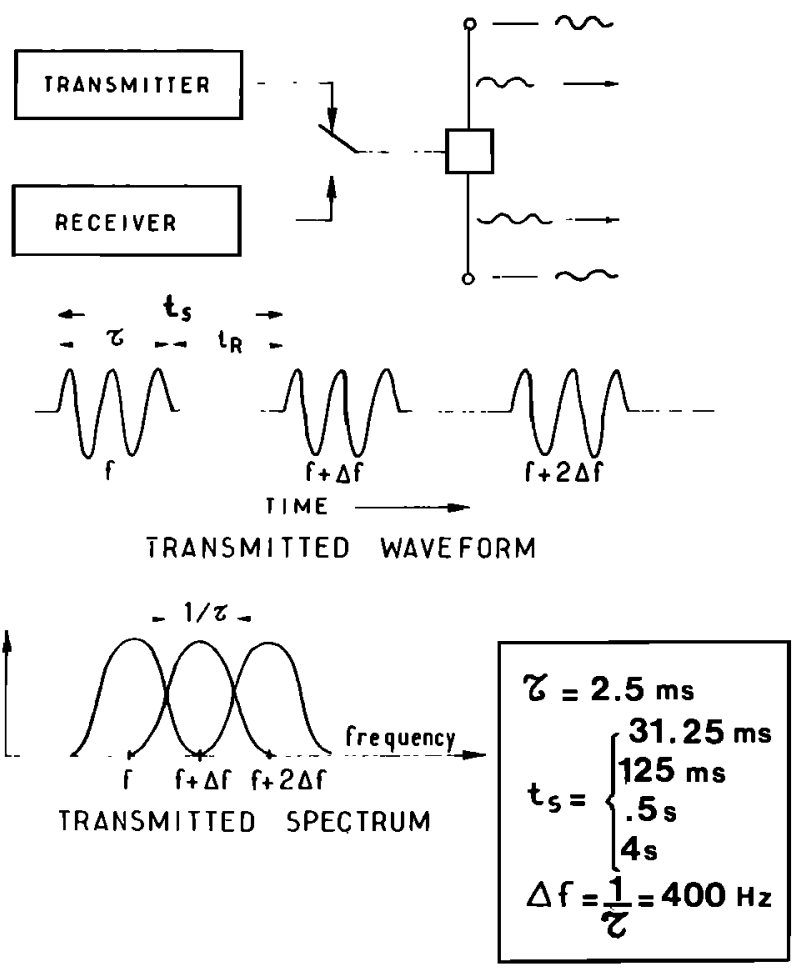


ISEE 1 December 8,19770322

$26.5 \mathrm{kHz}$
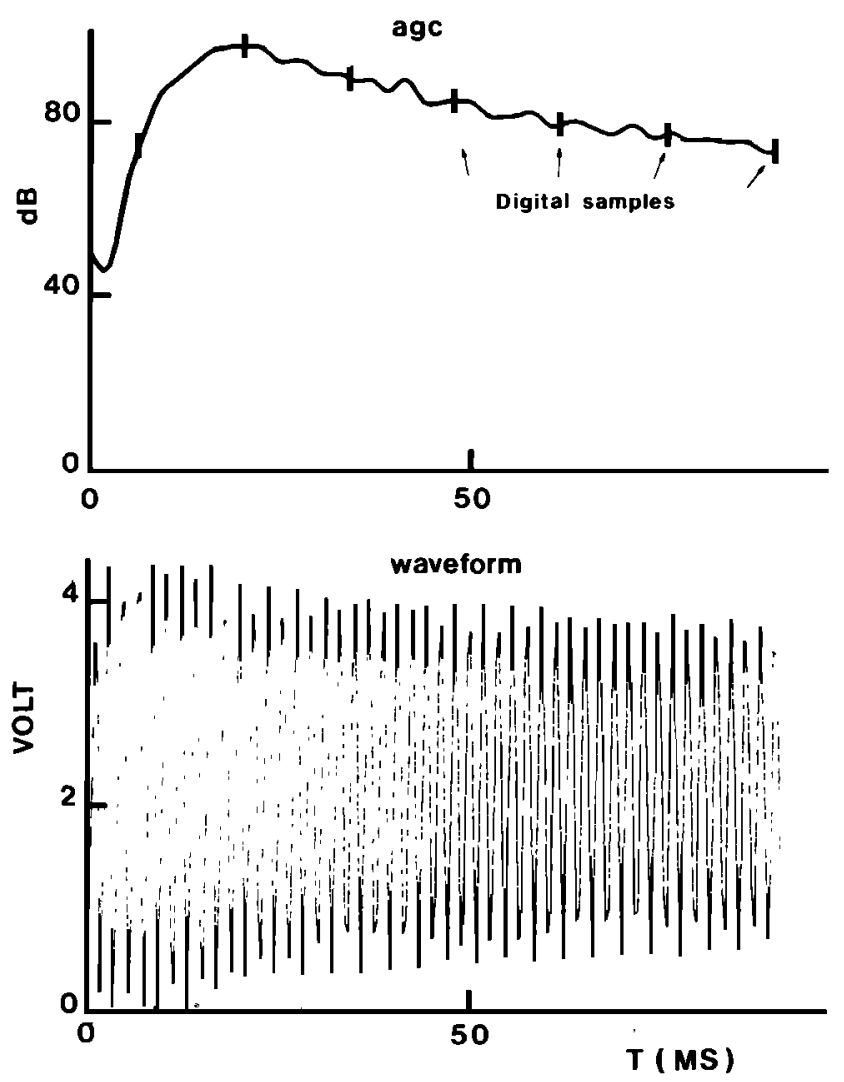

Fig. 2. Resonance in the solar wind. Top: envelope of the received signal in logarithmic scale. Bottom: compressed waveform. A resonance is a strong, monochromatic signal decreasing with time.

As a result of the ellipticity of the ISEE 1 orbite (apogee 23 $R_{E}$, perigee $280 \mathrm{~km}$ ), the sounder makes measurements in different plasma regimes (magnetosphere, magnetosheath, solar wind, magnetospheric tail) in which the signals have very different signatures. Consequently, we developed different methods to determine the plasma frequency, and thence the plasma density, in different regions.

We will first describe briefly the principle of the relaxation sounder and the signals observed in different regions. In sections 3 and 4 we will detail the method used in the regions (magnetosheath and solar wind) where only one resonance is observed at the plasma frequency. Section 5 will deal with the determination of the plasma frequency in the magnetosphere, where many resonances of different natures are observed.

\section{Relaxation Sounder: Resonances}

The principle of a relaxation sounder is similar to that of a classical radar flown in a plasma (Figure 1). At the beginning of a time interval $t_{s}$, a radio wave transmitter is corihected to an antenna and sends a wave train with a center frequency $f$ and a duration $\tau$. Immediately after this transmission a radio receiver tuned to the same frequency $f$ is connected to the antenna; this receiver has a bandwidth $\Delta f=1 / \tau$ and listens to signals in the frequency band $f \pm \Delta f / 2$ until the end of the time $t_{s}$ (frequency step). Then the frequency is incremented by $\Delta f$ and the process is repeated at $f+\Delta f$ (new frequency step).
Let us notice that the receiver bandwidth $\Delta f$ of the topside sounders is much larger than $1 / \tau$. The reason is that the receiver of this type of sounder sweeps upward in frequency during the travel time of the pulse [Franklin and Maclean, 1969]. The ISEE 1 relaxation sounder has many possible working modes chosen by telecommand [Harvey et al., 1978], but the one most commonly used covers the frequency range from 0 to $51 \mathrm{kHz}$ using 128 frequency steps, each of which is $400 \mathrm{~Hz}$ wide. The step duration $t_{s}$ is $125 \mathrm{~ms}$, a complete cycle lasting thus $16 \mathrm{~s}$. The received signal is compressed in an automatic gain control amplifier (AGC). The data are transmitted to the ground (1) either through a digital telemetry giving the AGC level, usually sampled once every $16 \mathrm{~ms}$, which is used permanently (the plasma frequency determination will be made on these signals) (2) or through an analog telemetry, used only $10 \%$ of the time, which transmits both the AGC level and the compressed waveform (these data will be used to help in the adjustment of the data processing).

If the transmitter is not used (passive mode), the sounder becomes a natural wave receiver. The sounder is usually active

RESONANCES IN THE SOLAR WIND (1290)

( RAW VARIABLES)

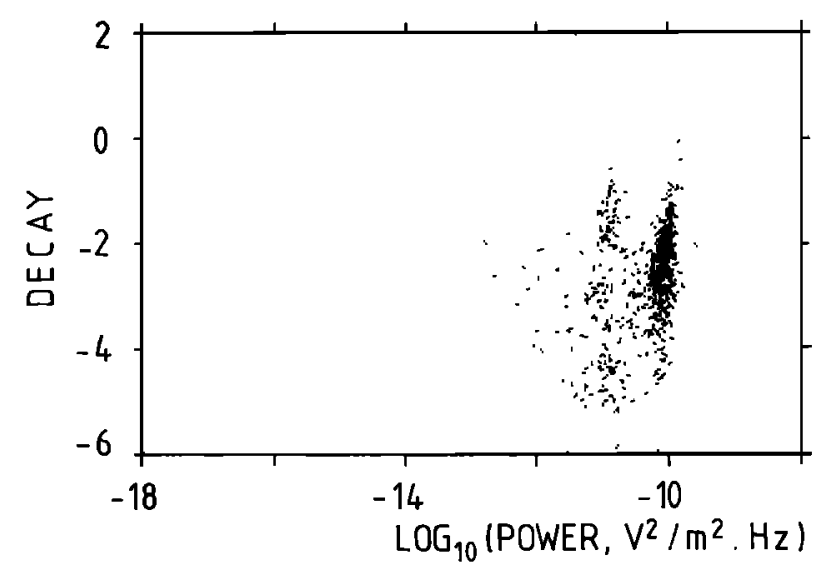

NON-RESONANCES (127800)

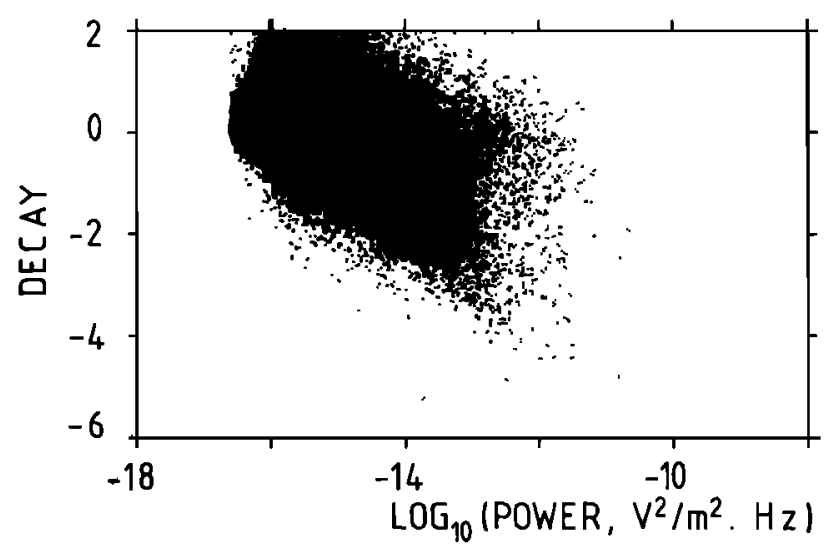

Fig. 3. Scatter plots of the raw variables in the solar wind for resonances (top) and nonresonances (bottom). The scattering inside one family is larger and the decoupling between the two families is less clear than for the more sophisticated variables of Figure 5. 


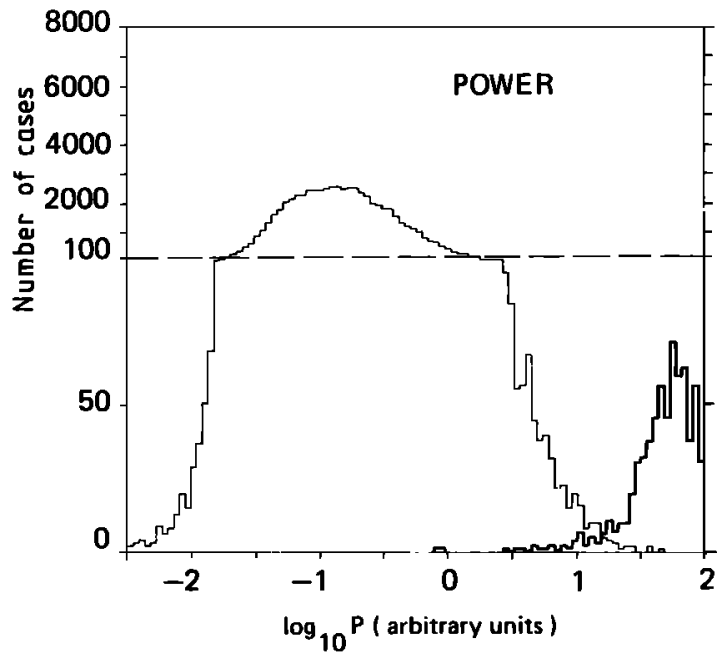

\section{ISEE 1 \\ November 6 to II. 1977}
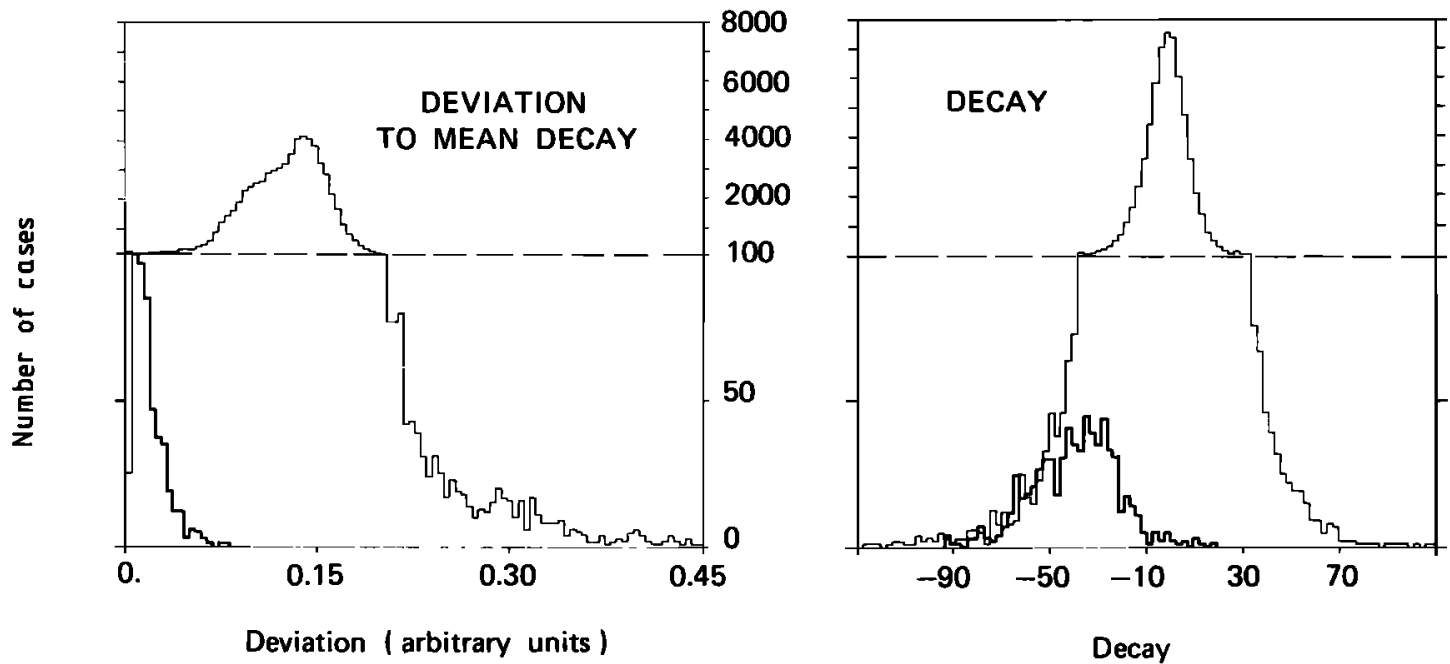

Fig. 4. Characteristics of resonances (thick line) and nonresonances (thin line) in the solar wind: "power" (top), "deviation" to mean decay (bottom left) and "decay" (bottom right). Resonances and nonresonances are well decoupled, especially for "power."

only part of the time, but in this paper we will deal only with data recorded during active periods.

The answer of the medium to the transmitted wave train, observed only at some frequencies, is called a resonance. Immediately after transmission, a very intense signal is observed, whose amplitude decreases slowly compared to the duration of the transmission $\tau$ : a resonance lasts approximately one hundred times more than the transmitted pulse. Such a phenomenon can only be observed when the frequency of the transmitted signal is close to a resonance frequency of the medium at which the group velocity of the waves is low, between one and a few tens (depending on the type of resonance observed) of times the relative velocity of the satellite with respect to the medium. In this case, the transmitted waves travel with the spacecraft, or intercept its trajectory, in such a way that the received signal lasts much longer than the initial pulse.

Detailed interpretations have been given for observations made in the ionosphere by Deering and Fejer [1965], McAfee [1969], and Fejer and $Y u$ [1970]. For the sake of the present study we will only mention that in the magnetosphere many resonances are observed: at the harmonics of the electron gyrofrequency, at the $f_{q n}$ frequencies of the Bernstein's modes of propagation, at the upper hybrid frequency [Etcheto et al., 1981]. It has been shown [Belmont, 1981] that in a biMaxwellian plasma (made of a dominant cold plus a hot population) there is a series of $f_{a n}$ (one in each branch contained between two successive gyroharmonics above the upper 


\section{RESONANCES IN THE SOLAR WIND (690) (AUTOMATIC RECOGNITION)}
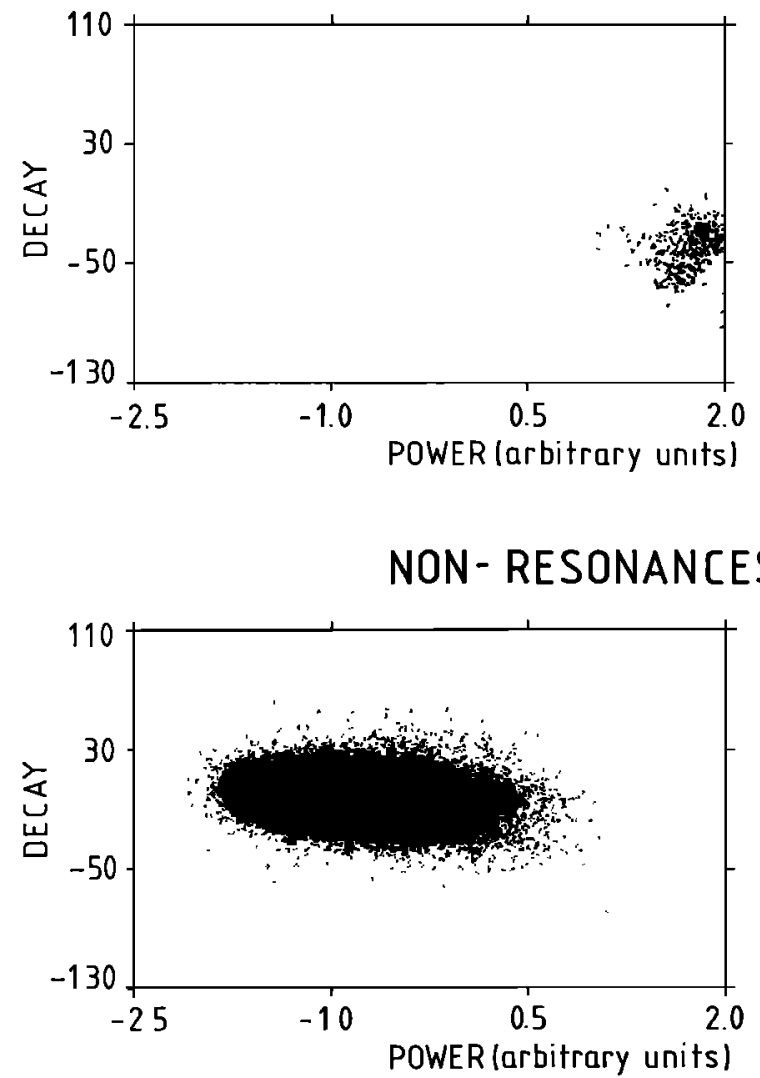

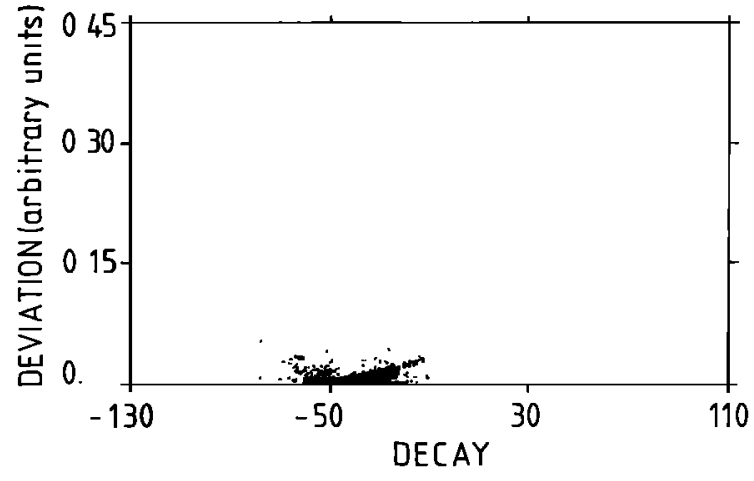

IN THE SOLAR WIND (86000)

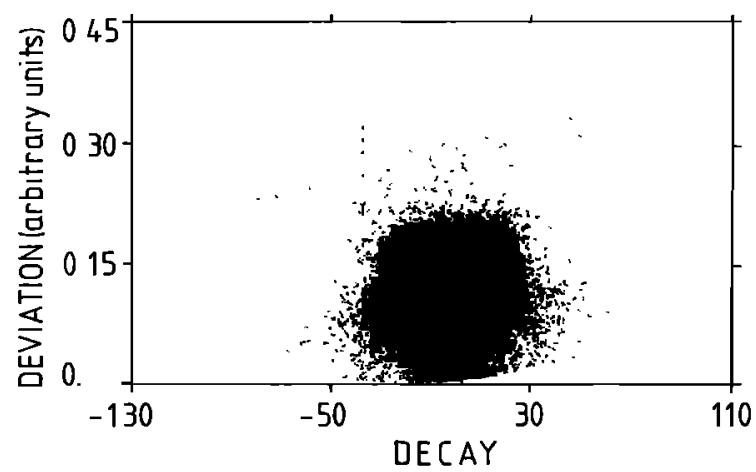

Fig. 5. Scatter plots of the variables for resonances (top) and nonresonances (bottom) in the solar wind. The decoupling is even clearer in a three-dimensional space.

hybrid frequency) which only depend on the ratio of the plasma frequency of the coldest population to the gyrofrequency and on the number of the branch in which they are observed. We will use this property later. Furthermore, additional $f_{q n}$ resonances, called "hot $f_{q n}$," are observed whose number and frequencies depend on the characteristics of the hot plasma. The intensity of the various resonances varies but usually the strongest signal is observed close to the total plasma frequency [Etcheto et al., 1983].

The data processing of the resonances in the magnetosphere will consist in determining the gyrofrequency (using the harmonicity of the gyroharmonics) and the cold plasma frequency (through the alignment of the cold $f_{q n}$ ) [Trotignon et al., 1982]. In the solar wind and magnetosheath where the electron temperature and the magnetic field are low, only one strong, longlasting resonance is observed close to the plasma frequency, even though an additional series of weak, short-lived resonances at the gyroharmonics and $f_{q n}$ is seen in the magnetosheath. In these regions, the data processing will consist in signal pattern recognition to identify the plasma resonance [Thouvenin and Trotignon, 1980], which is not more than one or two percent off the plasma frequency as determined by manual scaling of the data [Etcheto et al., 1981].

\section{SOLAR WIND}

A typical example of plasma resonance observed in the solar wind is shown in Figure 2, transmitted through the analog telemetry. At the top the AGC level is displayed as a function of time, the times of sampling of the digital telemetry being indicated. It is a strong signal, decreasing slowly with time. At the bottom, the compressed waveform (after frequency transposition) is plotted: its amplitude evolves slightly because the feedback is not total (the waveform is not compressed to a fixed amplitude but is only compressed from a 0 to 80-dB dynamic range to a 0 - to 20-dB dynamic range). We will work on the digitized AGC signal, usually sampled 3 or 7 times during a given frequency step.

From now on, we will call resonances the frequency steps where the observed signal behaves like a resonance, and nonresonances all the other steps. Our problem is now to separate the resonances from the nonresonances, automatically by using a computer, working on undersampled, possibly noisy signals, and keeping in mind that natural waves with comparable intensity can be present simultaneously. This task is well performed using a pattern recognition technique. As the resonances have a common pattern, while the nonresonances can be anything (natural waves of various kinds, parasitic signals), we are only able to define the common features of the resonances. These features will characterize the class of resonances. Then we have to determine whether a given frequency step belongs to the class of resonances or not by a comparison of its features with the already known pattern of this class. Among the pattern recognition techniques, we have chosen Sebestyen's algorithm, which seemed to be the most suitable in 


\section{ISEE 1 November 6 to II, 1977}

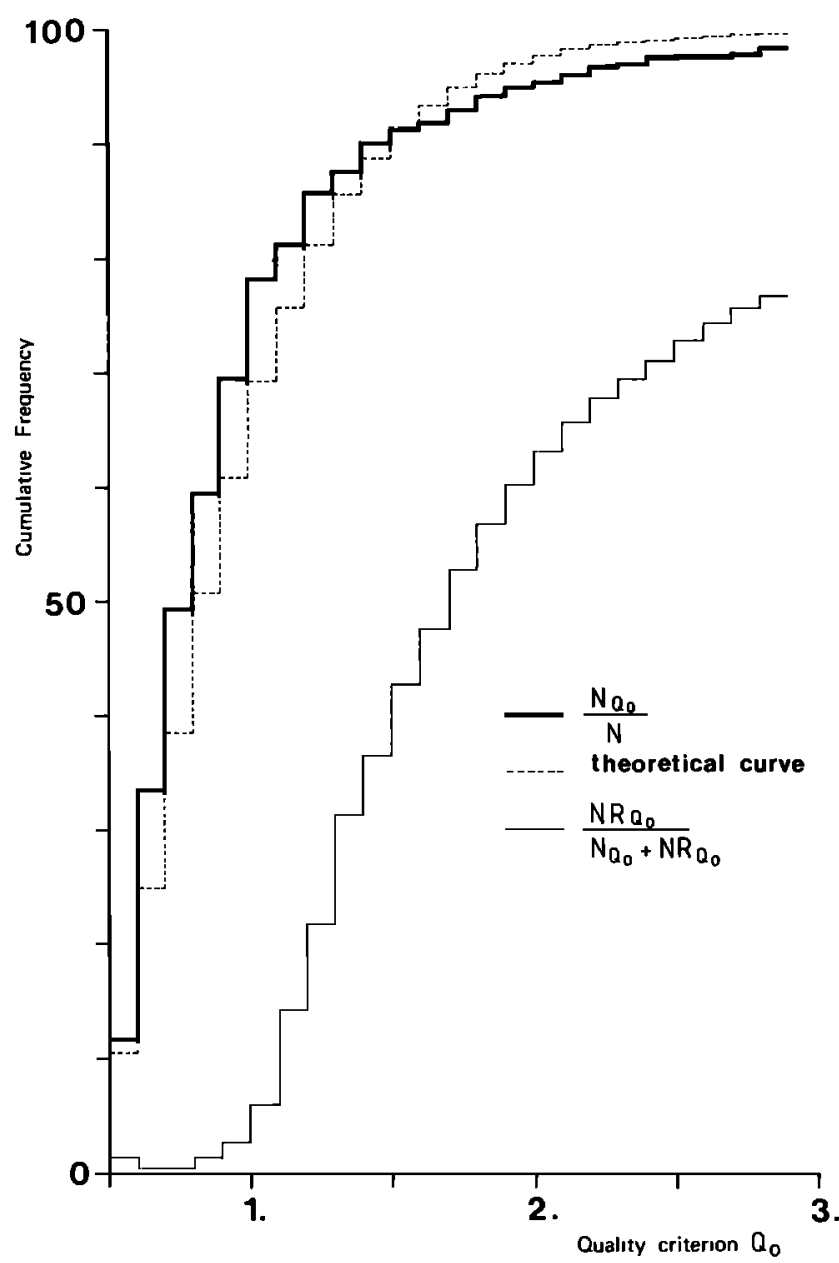

Fig. 6. Percentages of signals having a quality better than a given $Q_{0}$ for resonances (thick line) and nonresonances (thin line). The dotted line shows the theoretical results if the variables were normal. $N$ is the total number of resonances $(N=690) . N_{Q 0}\left(N R_{Q 0}\right)$ is the number of resonances (nonresonances) with $Q \leq Q_{0}$. We must improve the recognition.

our case (see Sebestyen [1962], Romeder [1973], and Thouvenin and Trotignon [1980] for its application to this particular problem).

First we must find the common pattern of the resonances. Through a measurement process which is done on a reference set of resonances we obtain a set of numbers called variables which best describe a resonance. Two "raw variables," named "power" and "decay," may be used. The "power" is the maximum power of the signal on the frequency step in $(\mu \mathrm{V} / \mathrm{m})^{2}$ $\mathrm{Hz}^{-1}$ without removing the noise of the instrument, and the "decay" is the slope $A$ of the signal assuming a time variation of the form $t^{A}$ (the definition of $A$ is given in the appendix). Figure 3, for which nearly 1300 frequency sweeps were used, shows the scatter plots of the decay versus the decimal logarithm of power for the resonances (top) and nonresonances (bottom). Note that the average decay of the resonances is close to the value - 2.35 given by Etcheto et al. [1981] using the analog telemetry.

The set of points representing the resonances does not satisfactorily cluster in the sense that the "distances" between the points are not small enough, on average. Moreover the cluster of resonances is not well separated from the cluster of nonres- onances. The fingerlike structure and spread of the clusters originate from the various modes in which the experiment was working (different sampling rates and times, change of the time constant of the AGC). It is worth noticing that the nonresonances decay with time. This is the result of a spurious signal, due to the transmitter, observed at the beginning of each step; the stronger the interference, the larger the decay, giving a slope to the cluster of the nonresonances. These are the reasons why we had to use the more sophisticated variables described in the appendix.

Using these three variables called "power," "decay" and "deviation" we will consider that the resonances are represented geometrically as points in a three-dimensional space. The first task of Sebestyen's algorithm is then to transform this original space in order to increase the clustering of these points. In the new space the mean-square distance $D^{2}$ between the members of the reference set of resonances is minimum (for more details see Sebestyen [1962]). In Figure 4 are shown the histograms of the new variables which are obtained after having done the transformations which minimize $D^{2}$. For the reference set of resonances that we used, the cluster is only rotated through an angle of nearly three degrees about the deviation axis. Therefore the original variables (defined in the appendix) are very nearly independent. These histograms are made out of 690 frequency sweeps (containing 690 resonances and $690 \times 128=88,320$ steps). We excluded the known parasitic lines at 20 and $40 \mathrm{kHz}$ [Harvey et al., 1979] which are usually as strong as the resonances but without any decay. The histogram for the resonances is a thick line while it is a thin line for the nonresonances. The horizontal dashed line indicated the change from an enlarged linear scale to a more compressed one. The power is by far the most discriminating variable, followed by the "deviation." The decay comes last. Tests made when sorting the resonances by frequency bands did not show appreciable modifications.

We displayed the variables separately but the computer will handle them simultaneously and work in a three-dimensional space, which representation is difficult. Thence we will project the two clusters (of resonances and nonresonances) on the planes orthogonal to the axes. The scatter plots in the "decaypower" (left) and "deviation-decay" (right) planes are shown in Figure 5 for the resonances (top) and nonresonances (bottom). This time the resonances cluster well, and the cluster of resonances (less than $1 \%$ of the observed signals) is well separated from the cluster of nonresonances: the chosen variables describe properly the resonances, and the discrimination is satisfactory.

Keeping in mind that the problem of finding the common pattern of the resonances is tackled by performing the transformations of the initial space which will cluster most highly the resonances in the new space, we can say that the calculation of $D^{2}$ is a mathematical way to express this common pattern.

We are now dealing with the second task of Sebestyen's algorithm, called the decision making process. We want to know whether a given frequency step $A$ belongs to the class of resonances or not. We must compute $S(A)$ the similarity of this signal to the signals of the class of resonances (here similarity means closeness in the new space). $S(A)$ is the meansquare distance between $A$ and the class at resonances represented by the set of points in the new space. For each frequency step that we wish to sort out we will calculate the normalized coefficient $Q(A)=S(A) / D^{2}$ which measures the quality of the signal pattern recognition: the smaller it is, the more likely is $A$ to be a resonance. This quality coefficient varies within the range $(0.5,+\infty)$. When the number of reso- 


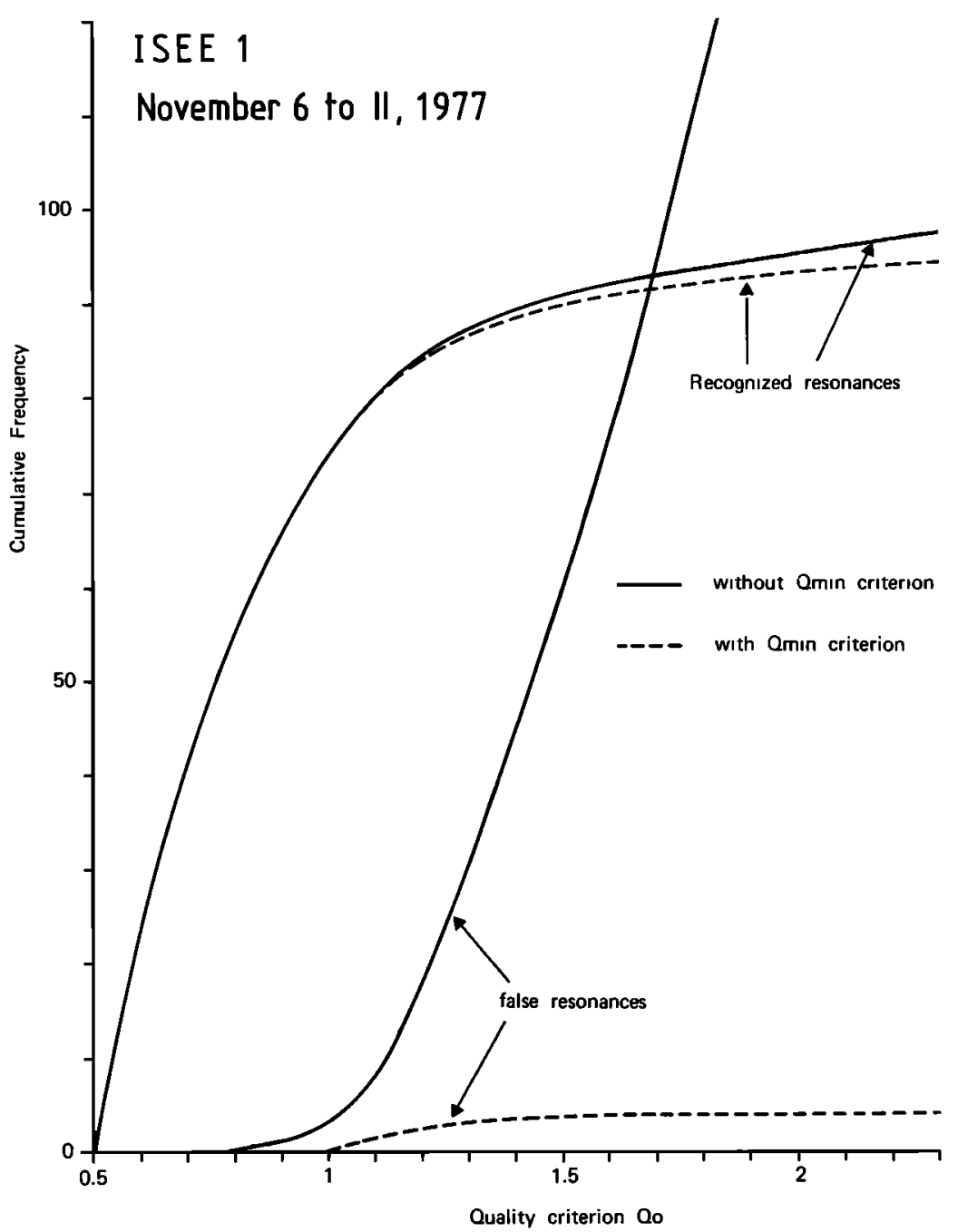

Fig. 7. Percentages of recognized resonances and false resonances versus $Q_{0}$ when taking all the signals with $Q \leq Q_{0}$ (solid line) and when taking only the signal with the best quality $\left(Q_{\min } \leq Q_{0}\right)$ in each sweep (dashed line). This latest test does not change much the number of recognized resonances but decreases drastically the number of false resonances.

nances of the reference set tends to infinity, the lower bound is reached at the gravity center of the class of resonances, and the average value of $Q$ for the set of resonances is 1 .

We will now discuss some results. Let us consider $N$ frequency sweeps in which we have identified the resonances by a manual process. Each sweep is made of $K$ frequency steps and only one is a resonance. For each step of the $N \times K$ steps under study we calculate its $Q$ quality coefficient, and then for a given threshold $Q_{0}$ we look for the $M_{Q_{0}}$ steps having $Q \leq$ $Q_{0}$. Among these $M_{Q_{0}}$ steps, $N_{Q_{0}}$ are really resonances while $N R_{Q_{0}}$ are nonresonances $\left(M_{Q_{0}}=N R_{Q_{0}}+N_{Q_{0}}\right)$. The results of this process are plotted in Figure 6. The percentage $N_{Q_{0}} / N$ of true resonances satisfying $Q \leq Q_{0}$ is drawn against $Q_{0}$ with a thick line. For comparison we have plotted in dotted line the theoretical curve under the assumption of normal variables. Moreover, to evaluate the pollution rate of the recognition process, the percentage $N R_{Q_{0}} / M_{Q_{0}}$ of nonresonances with $Q \leq Q_{0}$ against $Q_{0}$ is shown by a thin line. Among the steps with $Q \leq 1.3,78 \%$ are resonances (which represent $86 \%$ of the resonances of the reference set) and $22 \%$ nonresonances. For a threshold of $1.7,92 \%$ of the resonances are recognized but an equal number of nonresonances are taken with them, while for $Q \leq 1$ there is a $3 \%$ pollution but only $70 \%$ of the resonances are recognized.
If we now assume there is, at most, one resonance per frequency sweep, we can improve considerably the results. Of course, when the plasma frequency is out of the range of the sounder (obviously, there is no such case in the reference set), the program will give a false resonance. Fortunately this "resonance" will usually have a bad quality coefficient and will be rejected through using an adequate threshold value of $Q$. Moreover we do not take into account the case when the plasma frequency varies at a rate comparable with the frequency sweep and is observed several times. Therefore, in each frequency sweep we look for the step in which $Q$ is minimum and retain it as the most probable resonance. The results are shown in Figure 7 where the percentages of recognized resonances and false resonances having $Q \leq Q_{0}$ are shown versus $Q_{0}$, by dashed lines when using this $Q_{\min }$ criterion and by solid lines when not using this criterion. Since all these percentages are evaluated with respect to the total number $N$ of resonances which are expected, we can meet percentages of nonresonances $N R_{Q_{0}} / N$ greater than $100 \%$ when not using the $Q_{\text {min }}$ criterion (the nonresonances are more numerous than the resonances). When using the $Q_{\min }$ criterion a few resonances are lost $\left(1 \%\right.$ for $Q_{0}=1.7$ and $3 \%$ for $\left.Q_{0}=2.3\right)$ but the percentage of nonresonances is drastically decreased (zero for $Q_{0}=1,4 \%$ for $Q_{0}=1.7$ instead of $50 \%$ ). 


\section{RESONANCES IN THE MAGNETOSHEATH (1195)}

\section{( RAW VARIABLES )}

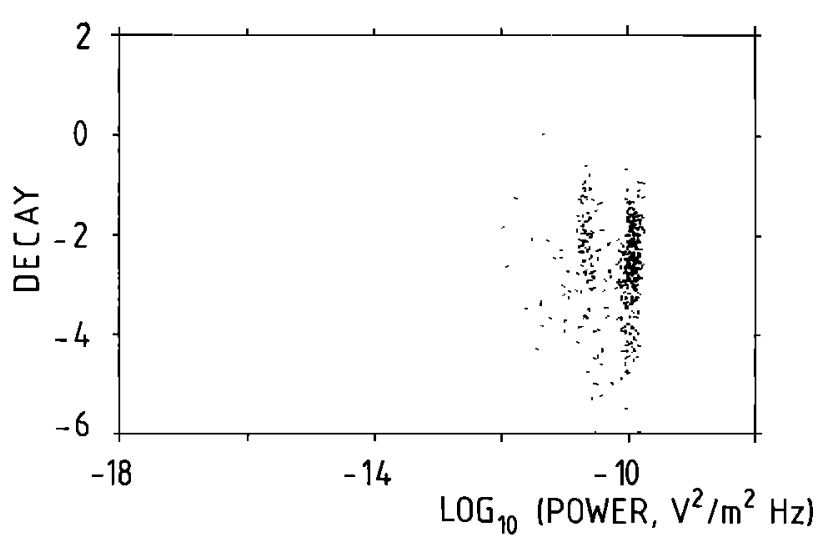

NON-RESONANCES (130600)

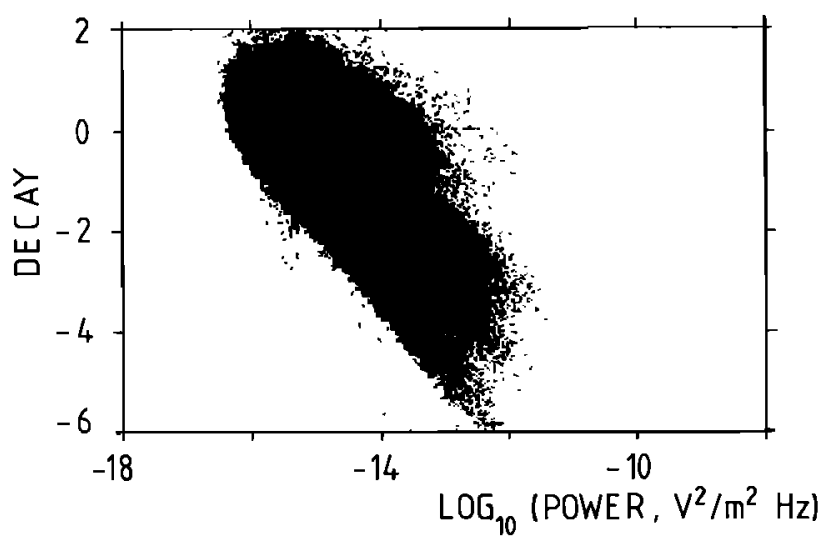

Fig. 8. Same as Figure 3, in the magnetosheath. The results are similar.

In summary, the steps with the minimum $Q$ value in each sweep will be $95 \%$ of the resonances and less than $5 \%$ of nonresonances when $Q \leq 2.5$. Depending on the way the results will be used, a threshold can be put on the $Q_{\min }$; for a low $Q_{0}$ some resonances will be missing but the recognized ones will not be polluted (that means, for a few sweeps there will be no recognized resonances). The accuracy of the plasma frequency determination is on the order of $400 \mathrm{~Hz}$ (one frequency step), that is, $4 \%$ for the density when the plasma frequency is $20 \mathrm{kHz}$. These results were corroborated by one year of data in the solar wind without any significant deviation of the characteristics of resonances with time.

\section{Magnetosheath}

In this region, as in the solar wind, only one strong, longlasting resonance is observed at a frequency close to the plasma frequency. At frequencies above the plasma frequency, additional, weak, short-lived resonances are observed at the harmonics of the electron gyrofrequency [Etcheto et al., 1981]. Since the gyrofrequency is on the order of $500 \mathrm{~Hz}$, these resonances are properly observed only using the analog telemetry and will be ignored, so that we will consider that only one resonance is observed at the plasma frequency.

The scatter plots of the decay versus power (raw variables) are shown in Figure 8 for resonances (top) and nonresonances (bottom) from about 1200 frequency sweeps recorded from December 29, 1977, to January 25, 1978. This figure should be compared to Figure 3 on which are displayed the same variables in the solar wind. The plots for the resonances are very similar, and the same signals pattern recognition method should be applicable. More precise comparisons made on the three variables power, decay and deviation showed no significant difference between plasma resonances in the solar wind and those in the magnetosheath. As a matter of fact, when Sebestyen's algorithm is used on the magnetosheath data, we obtain the same percentage of success using the solar wind reference set or the magnetosheath one.

At last we should point out a tendency for the power of the resonances to increase with frequency, as can be seen in Figure 9. Here the average power is plotted against frequency (raw variables) by bins of four steps, for both magnetosheath and solar wind resonance sets together. This is in line with the theoretical results of Deering and Fejer [1965] and Fejer and $Y u$ [1970] which predict a variation proportional to $f^{9 / 2}$ for signals accompanying the satellite or to $f^{7 / 2}$ for oblique echoes. These two laws are shown as solid lines in Figure 9 but the frequency range covered is too small and the receiver saturation level too low to decide between them.

We will now look at the results given by this program for a case of multiple bow shock crossings. Figure 10 shows a dynamic spectrogram of the sounder data for a three-hour period during which the earth bow shock was crossed several times. The frequency scale is linear between 0 and $51 \mathrm{kHz}$ while the grey scaling depends on the strength of the received signals. The tick marks at the top of the frame indicate the times when the sounder was active. At the beginning of the period the spacecraft is in the magnetosheath, and the plasma frequency varies between 40 and $51 \mathrm{kHz}$, going out of the range of the sounder at $2048 \mathrm{UT}$. The satellite enters the solar wind at 2054 , and the plasma frequency stays around $30 \mathrm{kHz}$ until 2120 when the spacecraft crosses the bow shock again. In the magnetosheath, between 2120 and 2130 the plasma frequency is out of range. When the spacecraft enters the solar wind again at 2130 , the plasma frequency is $33 \mathrm{kHz}$. From 2148 to 2154 a burst of upstream natural noise [Etcheto and Faucheux, 1984] is observed. The satellite makes a new excursion into the magnetosheath, where the plasma frequency is above $51 \mathrm{kHz}$, from 2205 to 2218 and from then on stays in the solar wind. The program for automatic resonance recognition was run on these data, and the results are shown in Figure 11. The frequency of the recognized resonance when using the $Q$ minimum criterion is plotted as a function of time, using a symbol which depends on its quality: an asterisk for $Q$ less than 1 , a cross between 1.0 and 1.1 , an $\mathrm{L}$ from 1.1 to 1.3 and a circle above 1.3. As we kept one resonance per sweep, there is a result even when the plasma frequency is out of range, but during these periods the quality is poor (hardly an asterisk), and if we had put a threshold on $Q$ we would have kept only true resonances. For this day, we had only three AGC samples per step.

\section{Magnetosphere}

In the magnetosphere, contrary to what is observed in the solar wind, many resonances of different nature are observed, and the problem is no longer to distinguish a resonance among nonresonances but to name the different resonances and deduce the plasma frequency. An example is shown in Figure 12 where the decay (top) and power (bottom) computed as raw variables on each of the 128 frequency steps are 


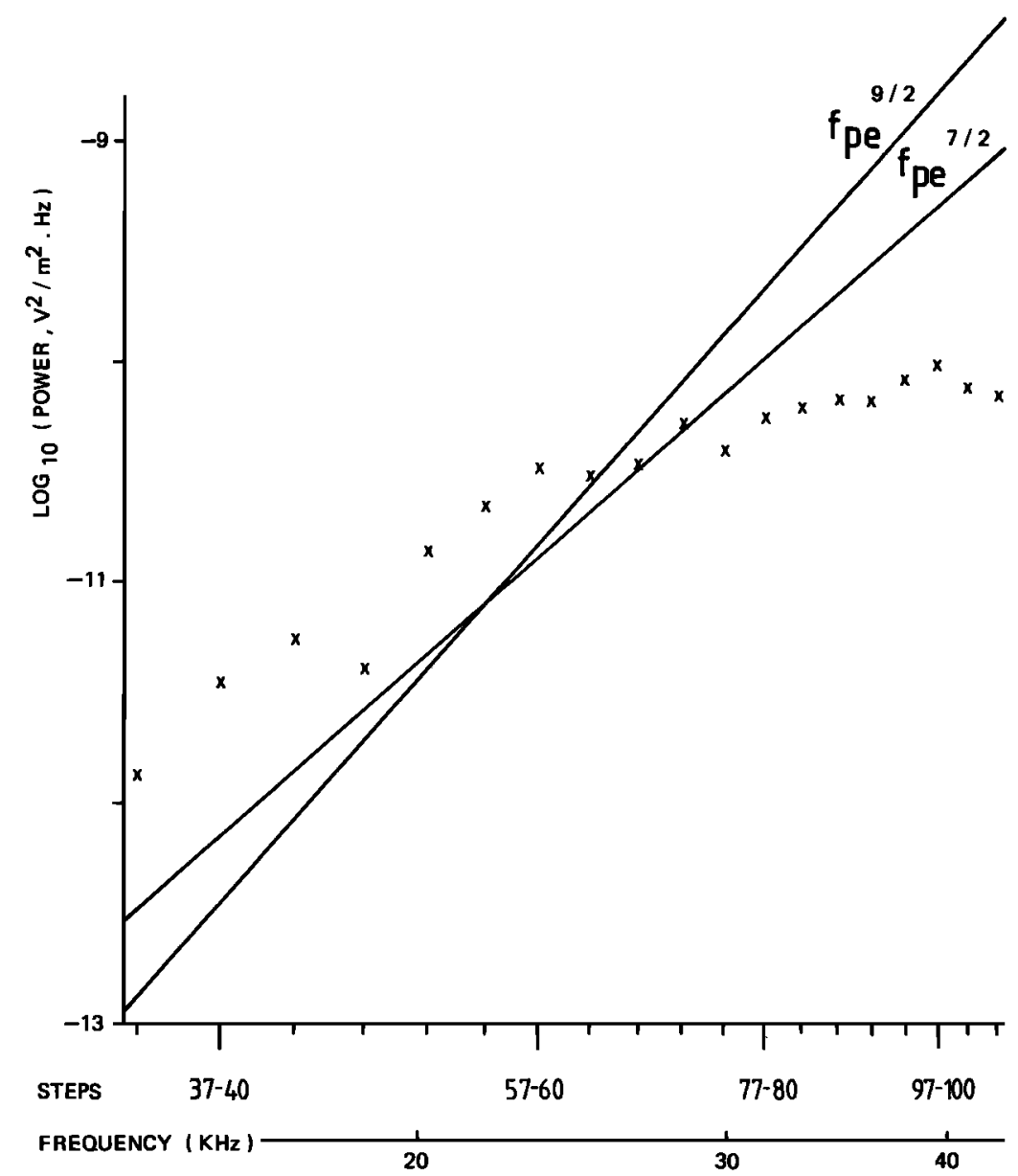

Fig. 9. Average power as a function of frequency for resonances in solar wind and magnetosheath. The power increases with frequency.

shown on a frequency versus time display, the grey scaling depending on the value of the parameter. During this period the satellite was at high magnetic latitude $\left(50^{\circ}\right)$, its geocentric distance varying from 5 to $7 R_{E}$ near noon. Two series of resonances due to propagation of the waves on the Bernstein's modes [Bernstein, 1958] are seen: the gyroharmonics $n f_{c e}$ and the $f_{q n}$ frequencies.

\subsection{Pattern Recognition Study}

The power and decay of the resonances are much more variable in the magnetosphere than in the solar wind. This can be checked in Figure 13 (which should be compared to Figure 3 ) in which the scatter plot of decay against power is shown for the resonances (top) and nonresonances (bottom) for a hundred sweeps recorded between November 29, 1977, and December 14, 1977. The two clusters are not far apart enough to allow for a proper discrimination. The reason is that many weak and short resonances resemble nonresonances and also that strong resonances give images on the adjacent steps, which are nevertheless considered as nonresonances.

The next question is, is it possible to sort out the various types of resonances using the raw variables power and decay? The bottom panels in Figure 14 show that the answer is again no. In this figure are plotted the power (left) and decay (right) for the resonances and nonresonances (top) and for the two types of resonances (bottom). The top panels confirm what is seen in Figure 13: the resonances and nonresonances are not sufficiently discriminated. In addition, we do not know in advance the number $N$ of resonances excited during one sweep, and it is not possible to select for instance the $N$ resonances having the best quality, a method which we have seen to be very efficient in the solar wind.

Therefore, in the magnetosphere the signal pattern recognition methods are not likely to give very good results, either to discriminate between resonances and nonresonances or to identify the different families of resonances. To quantitatively support these conclusions we have to show a comparison of the results obtained in the solar wind and in the magnetosphere by using a simple threshold criterion: $P \geq P_{0}$ and $D \leq D_{0}$, where the power $P$ and the decay $D$ are the raw variables defined in section 3. In Figure 15 we have plotted the percentage of resonances having $P \geq P_{0}$ and $D \leq D_{0}$ against the percentage of nonresonances satisfying the same criterion: for a given $P_{0}$ while $D_{0}$ is varying (solid lines) and for a fixed $D_{0}$ and $P_{0}$ varying (dashed lines). Note that those curves are respectively labeled in $\log P_{0}$ and in $D_{0}$. Moreover, the percentages are computed with respect to the total number of resonances which are contained in each data set (solar wind in the top frame or magnetosphere in the bottom frame). A data set means a group of sweeps in which we have identified all the resonances with a manual process. In these data sets the nonresonances are more numerous than the resonances, so for low values of $P_{0}$ and large values of $D_{0}$ we can have more nonresonances which satisfy the upper criterion than the total 


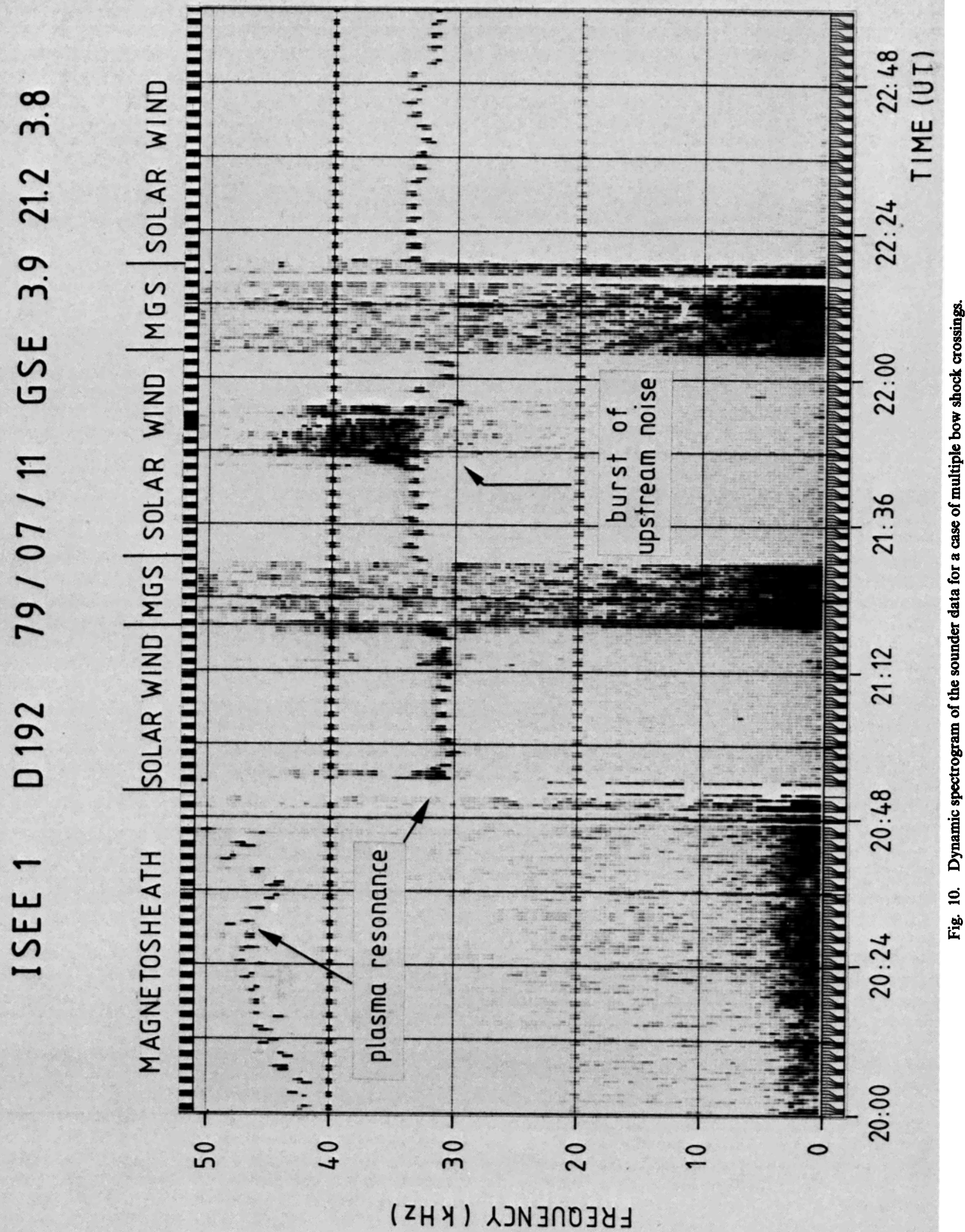


ISEE 1 D $19279 / 7 / 11$

* $\quad 0.5 \leqslant Q<1.0$

$L \quad 1.1 \leqslant Q<1.3$ $\times \quad 1.0 \leqslant Q<1.1$

- $1.3 \leqslant a$

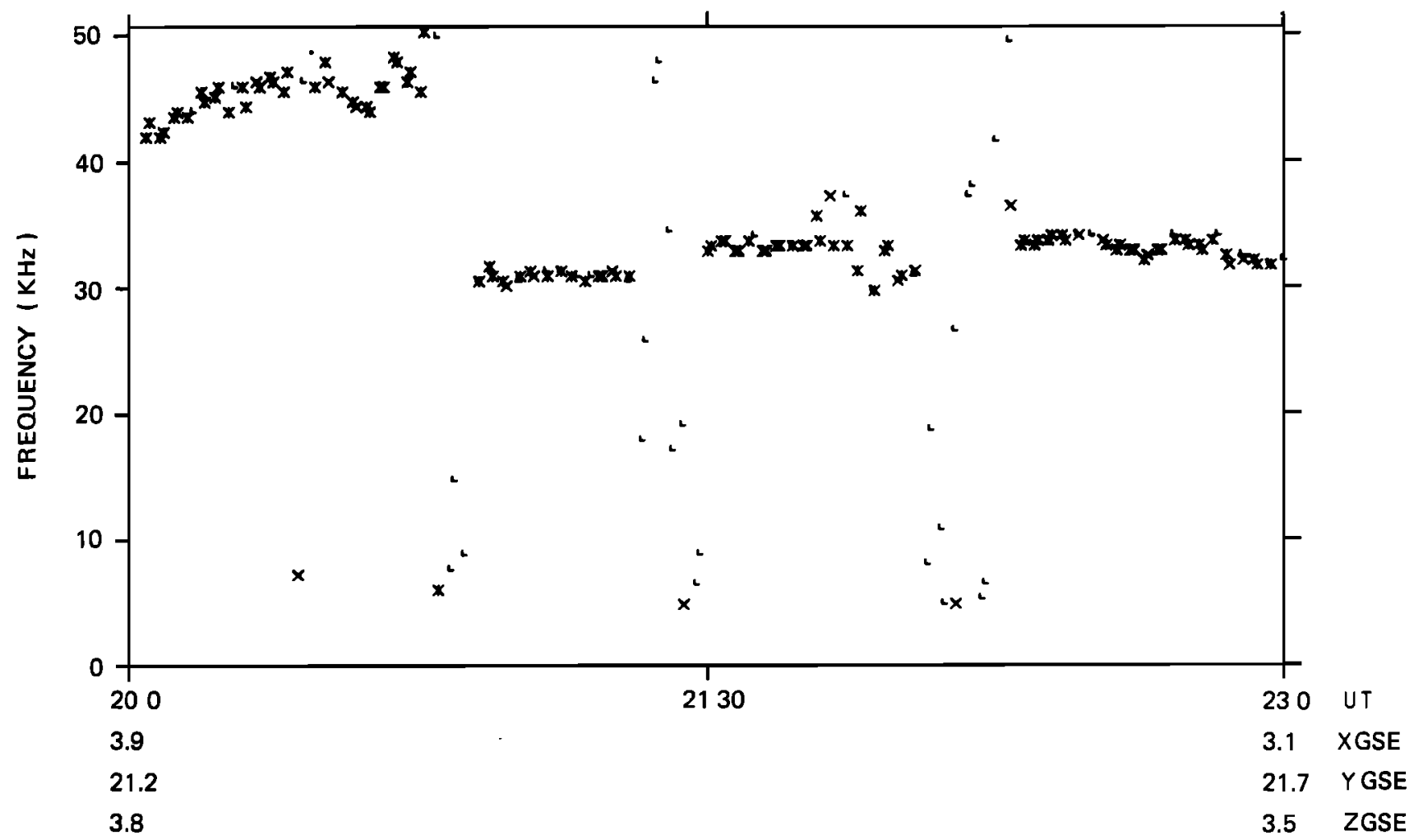

Fig. 11. Results of the automatic data reduction for the same period as in Figure 10. When the plasma frequency is out of the frequency range of the experiment, the quality of the most probable (false) resonance is bad.

number of resonances we are looking for. This is the reason why the percentage of nonresonances (PNR) can be greater than $100 \%$. To know the percentage of recognized resonances for given $P_{0}$ and $D_{0}$ thresholds one should consider the intersection of the relevant power and decay curves. Suppose that we have to sort out 100 resonances. If we accept a pollution of $20 \%$ by nonresonances (PNR $=20$ ), that is, if we do not accept more than 20 nonresonances among the recognized signals, we will not recognize more than 75 resonances $(P R=75)$ in the solar wind (which corresponds approximately to $\log P_{0}=-11.75$ and $\left.D_{0}=-1.8\right)$ and $65(P R=65)$ in the magnetosphere $\left(\log P_{0}=-12.5, D_{0}=-1\right.$.). If we allow only for $10 \%$ pollution, we will recognize 72 resonances in the solar wind and only 45 in the magnetosphere. Moreover, in the magnetosphere the average number of resonances per sweep is 30. So, if we accept a $20 \%$ pollution, we will obtain $30 \times 0.65=19.5$ good resonances and $30 \times 0.2=6$ wrong resonances per sweep instead of the 30 good resonances we were looking for. This is the reason why this recognition method (even improved by using Sebestyen's algorithm) is not usable in the magnetosphere and we will then rely on the physical knowledge that we have of the phenomenon.

\subsection{Use of Dispersion Relation} of Bernstein's Modes

Figure 16 shows the received amplitude (the average of the electric field) on the ordinate (after subtracting the noise of the experiment) as a function of frequency. In this display the resonances appear as peaks.

The most easily identified are the gyroharmonics, due to their harmonicity. We use the measurement of the on-board magnetometer as an initial value and compare the data to the series of resonances, computed in frequency steps for several gyrofrequencies taken close to this initial value. This processing is applied after removing from the signal the slow variation of the amplitude with frequency, which is determined in the way explained below. The best fit gives the gyrofrequency with an accuracy of $0.7 \%$ (the modulus of the magnetic field is known within less than $1 \gamma$ in a 100- $\gamma$ field).

We can now determine the $f_{q n}$ resonances and the plasma frequency $f_{p e}$. We have seen that in a Maxwellian plasma the $f_{q n}$ normalized to the gyrofrequency only depend on the plasma frequency (normalized to the gyrofrequency too). We use the dispersion relation, stored in the computer, to deduce series of theoretical $f_{q n}$ for various values of $f_{p e}$. We then compute the frequency of these theoretical resonances using the gyrofrequency that we have just determined. Each $f_{q n}$ series is then compared to the observed signals, and weighted in order to favor the lowest-order $f_{q n}$. The $f_{q n}$ series which best fits the observations gives at the same time the theoretical $f_{q n}$ and the plasma frequency. If the plasma is not Maxwellian, it will be the plasma frequency of the coldest component. But, in order to minimize the computing time of this correlation, we need a reasonable initial value. We have established from many observations in the magnetosphere that the resonances are more 


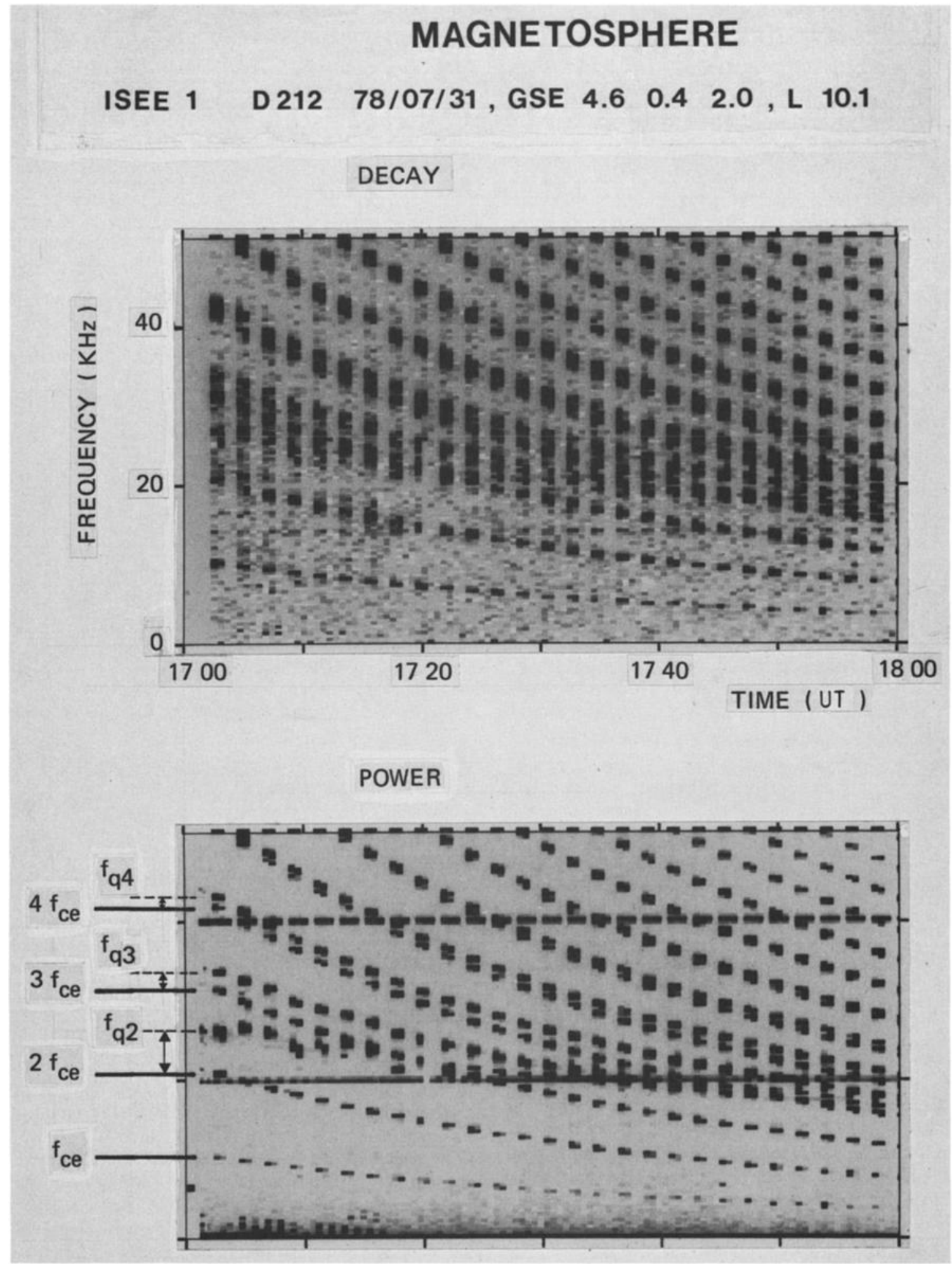

Fig. 12. Dynamic spectrogram of the raw variables, "decay" (top) and "power" (bottom) for an outbound pass in the magnetosphere. Many resonances are observed.

intense close, and above, the plasma frequency (a detailed study of one case observed on GEOS 2 is made by Etcheto et al. [1983]). We therefore smoothed the spectrum shown in Figure 16, obtaining the dotted curve, which frequency of maximum amplitude was used as the initial value of the plasma frequency. The smoothing is obtained by computing the fast Fourier transform of the signal made of the 128 values measured in one sweep and filtering this spectrum by multiplying by $[1+\cos (\pi J / 10)] / 2$ the ten lowest-frequency components $(J=0,1, \cdots, 9)$ and by 0 the higher-frequency components. This filtered spectrum then undergoes the inverse fast Fourier transform, resulting in the dotted curve of Figure 16. 
RESONANCES IN THE MAGNETOSPHERE (2640)

(RAW VARIABLES)

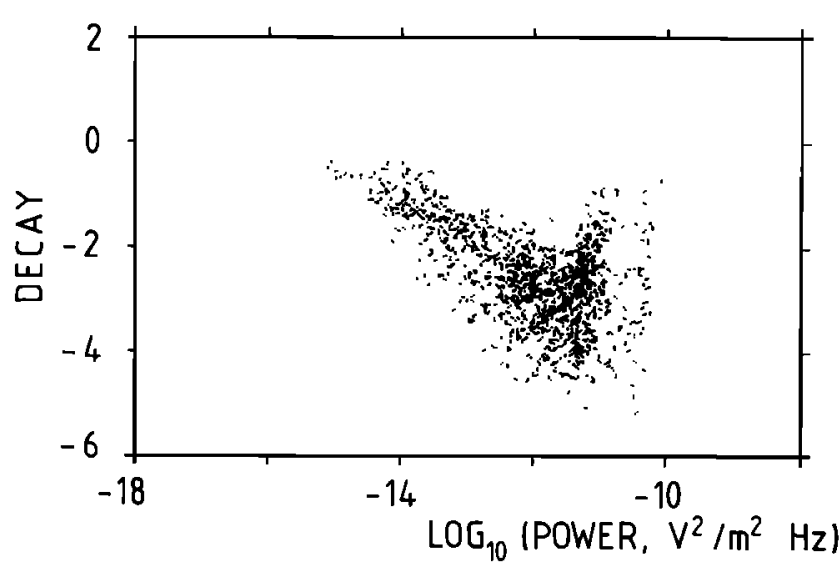

NON-RESONANCES (10200)

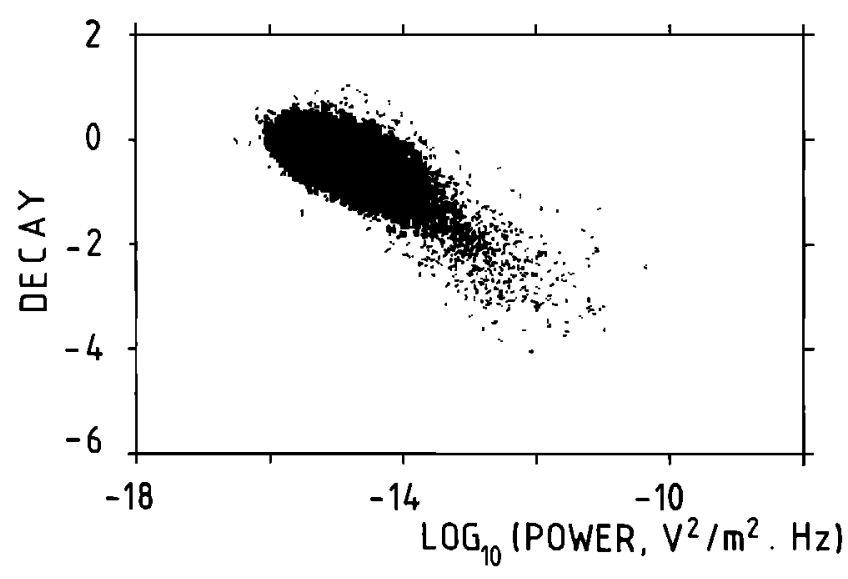

Fig. 13. Same as Figures 3 and 8 in the magnetosphere. The resonances represent now $20 \%$ of the signals and are no longer clearly decoupled from nonresonances.

Above the curves of Figure 16 is shown the result of the identification of the resonances. At the top the results of the manual identification (using the analog telemetry) are gyroharmonics, $f_{q n}$, and $f_{x}$, which does not belong to these two families. Below, the results of the automatic analysis are as described above. The gyroharmonics and $f_{q n}$ are correctly recognized (with $400-\mathrm{Hz}$ accuracy). The plasma frequency $f_{p e}$ deduced from the $f_{q n}$ resonances does not correspond to a resonance and is shown above $3 f_{c e}$ by a thick arrow.

In order to discuss in more detail this method for determining the plasma frequency, using the $f_{q n}$ resonances, we will use Hamelin's diagram shown in Figure 17 [Hamelin, 1980; De Feraudy and Hamelin, 1978]. All the frequencies are normalized to the gyrofrequency $f_{c e}$. Each curve labeled $n$ is the locus of a given $f_{q n}$ when the plasma frequency varies. On the ordinate is plotted the deviation in frequency between this $f_{q n}$ and the gyrofrequency just below (of order $n$ ) versus the plasma frequency (plotted on the abscissa). For a given plasma frequency $f_{p e}$, all the $f_{q n}$ are aligned on a vertical line, provided the plasma is Maxwellian. If we identify an $f_{q n}$ resonance, knowing the gyrofrequency, we can mark it on this diagram. If several $f_{q n}$ resonances are on a vertical line, we can conclude that the plasma is close to Maxwellian and deduce the plasma frequency.

On the left part of Figure 17 we have plotted with horizontal lines the results of the manual identification using the analog telemetry ( $8-\mathrm{Hz}$ frequency resolution) and with thick lines the results obtained using the digital telemetry $(400-\mathrm{Hz}$ frequency resolution). The plasma frequency determined by the computer from the digital telemetry is shown by an arrow. Using the analog telemetry, each $f_{q n}$ resonance (except $f_{q 3}$ ) is split into two, but the agreement between the automatic determination of the plasma frequency and the manual determination is good, even when the analog telemetry is used.

On the right-hand side of Figure 17, another example is shown, in which the splitting of the $f_{q n}$ resonances is larger and the alignment less clear. Nevertheless the automatic recognition of the $f_{q n}$ resonances from the digital telemetry (thick lines) is still correct. Belmont [1981] has shown that in a nonMaxwellian plasma the $f_{q n}$ frequencies could be split and not aligned, corresponding to plasma frequencies ranging from the plasma frequency of the coldest component to the total plasma frequency. For a bi-Maxwellian plasma (a cool and a hot component), this range of apparent plasma frequencies is larger when the two populations are more decoupled. The splitting of the $f_{q n}$ that we usually observe on board ISEE 1 is of the type foreseen by the theory for two populations whose temperatures are not very different but whose densities differ significantly.

The program of automatic determination of the plasma frequency works on the digital telemetry and cannot see the splitting of the $f_{q n}$ resonances, owing to the lack of frequency resolution. It fits first the gyroharmonics (obtaining an average value when the gyrofrequency varies during the frequency sweep) and then uses this value of the gyrofrequency to compute the frequencies of the theoretical $f_{q n}$ resonances and fit them to the observations. The accuracy of this gyrofrequency has a strong influence on the fit of the $f_{q n}$ as can be seen on the inset in the center of Figure 17. A theoretical series of $f_{q n}$ lying on the vertical line at $f_{p e} / f_{c e}=4.4$ has been replotted on the Hamelin's diagram after normalizing it to $1.007 f_{c e}$ (left series) and to $0.993 f_{c e}$ (right series). The $f_{q n}$ are now clearly misaligned, more for larger $n$, to the left if the gyrofrequency is increased, to the right if it is decreased. This misalignment is still tolerable by the computer owing to the poor frequency resolution of the digital telemetry. A larger error for $f_{c e}$ would change the frequency of the first $f_{q n}$ by a quantity exceeding the frequency resolution of the experiment and is therefore not tolerable.

On the two Hamelin's diagrams of Figure 17 an additional resonance labeled $f_{x}$ (plotted with an asterisk) is observed between 3 and $4 f_{c e}$ in the left-hand diagram and between 4 and 5 in the right-hand one. It is likely [Belmont, 1981] that this resonance is close to the total plasma frequency while the alignment of the $f_{q n}$ resonances corresponds to the plasma frequency of the coldest population. One should notice that to look for the $f_{q n}$ resonance of lowest frequency and consider that the plasma frequency lies between this frequency and the gyroharmonic just below it, is risky. It assumes that it is possible to determine the first $f_{q n}$, which is not necessarily true, especially when it is between a critical value, on the order of $(n+0.7) f_{c e}$, and $(n+1) f_{c e}$, a region in which the damping is very strong when the propagation is not strictly perpendicular to the magnetic field [Pottelette et al., 1981]. The advantage of 


\section{MAGNETOSPHERE}
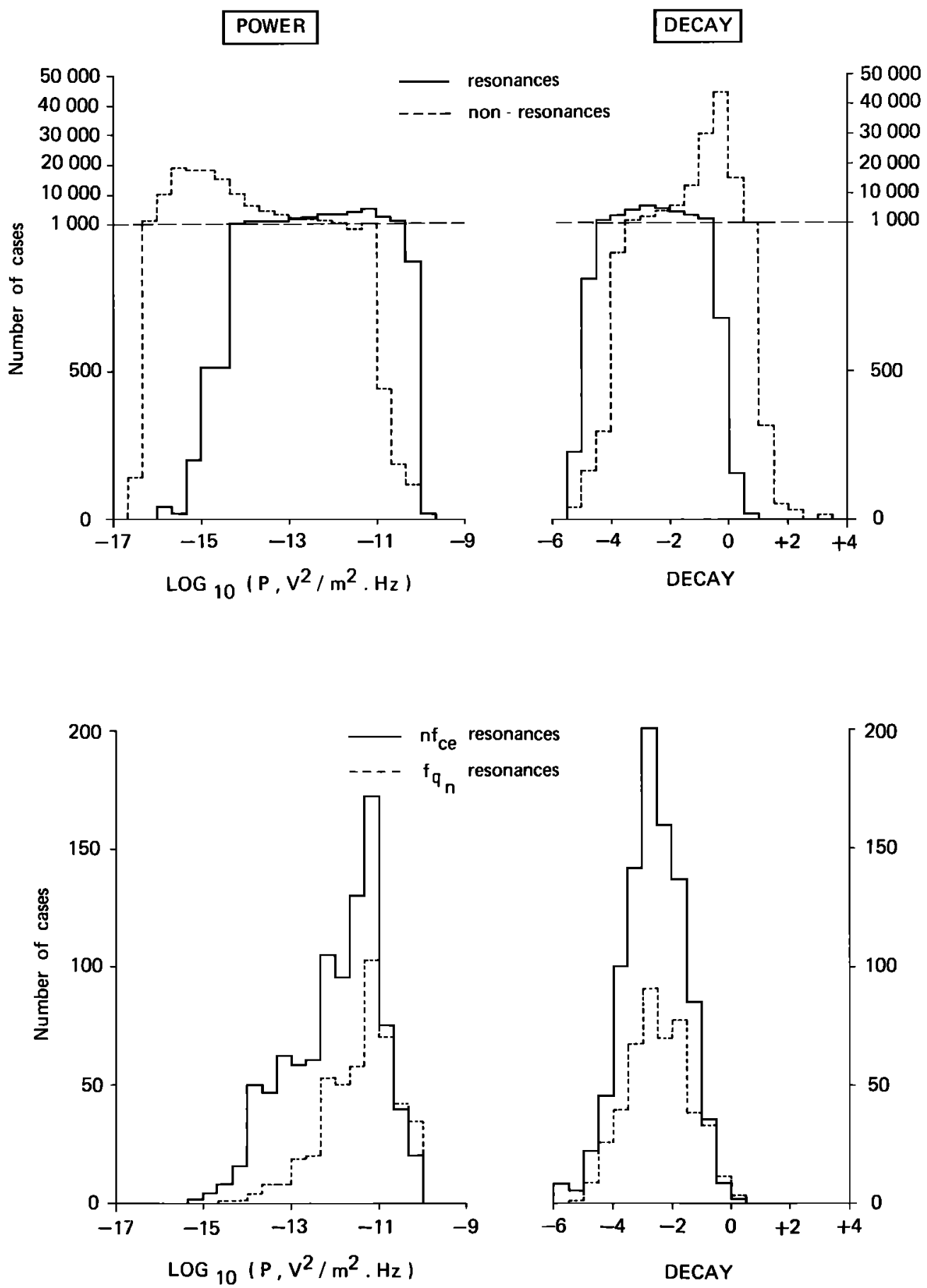

Fig. 14. Histograms of the raw variables "power" and "decay" in the magnetosphere for resonances (solid line) and nonresonances (dashed line) at the top and for gyroresonances (solid line) and $f_{q n}$ resonances (dashed line) at the bottom. The resonances are no longer decoupled from the nonresonances, and the different families of resonances have the same characteristics: the signal pattern recognition method has to be abandoned.

the technique of alignment of the $f_{q n}$ compared to this latest method is to use the whole set of $f_{q n}$, thus providing a necessary cross-check.

Out of 250 frequency sweeps that we studied carefully, the accuracy of the plasma frequency deduced by the computer from the $f_{a n}$ resonances is better than $10 \%$ in $93 \%$ of the cases. In $96 \%$ of the cases this frequency (which is close to the plasma frequency of the coldest population) is less than $10 \%$ lower than $f_{x}$ (which is close to the total plasma frequency). On the other hand, the frequency of the maximum of the 
smoothed spectrum (used as an initial value for the plasma frequency determination) is above $f_{x}$ in $97 \%$ of the cases. For $60 \%$ of the sweeps this maximum is less than $10 \%$ above $f_{x}$, and in $96 \%$ it is less than $20 \%$ above $f_{x}$. Usually this maximum is between the first and the second $f_{q n}$, and closer to the second one.

Figure 18 shows the dynamic spectrogram of the sounder data (continuously active), for an example of density fluctuations observed on January 13, 1982, between 1810 and 1910 UT, while ISEE 1 was leaving the plasmasphere. At low frequency only resonances at the gyroharmonics are observed. Above, the $f_{q n}$ are superimposed on the gyroharmonics, giving the impression of a darker region, whose lower edge corresponds to the plasma frequency. The results of the program of automatic determination of the plasma frequency are plotted as a function of time in Figure 19. In the top frame, the solid line is the density deduced from the alignment of the $f_{q n}$ while the dashed line represents the time evolution of the maximum of the smoothed spectrum. This latest curve is always above the density curve and follows the density fluctuations. In the bottom frame is displayed the modulus of the magnetic field after a rough detrending, obtained by substracting a mean magnetic field approximated by a polynomial of third degree. This rough processing is sufficient to evidence the compressional character of these Pc 5 pulsations. Even though the density is undersampled and the magnetic field averaged, the pulsations are in phase on both quantities (particularly at the beginning), as in the case studied by Kivelson et al. [1984] of a pulsation with a similar period of 7 minutes, but at a completely different local time (near noon). This pulsation is a quiet time event ( $K p \leq 1+$ during the previous 24 hours). Singer et al. [1979] studied Pc 3, 4 and 5 in the morning sector using simultaneous measurements on board several satellites, but their density measurements were not sampled fast enough to evidence fluctuations. Between 1815 and 1830 , superimposed on the pulsations, a density increase is observed simultaneously with a decrease of the modulus of the magnetic field, without any particular change in the direction of the magnetic field. This short-lived density increase could be due to a plasma cloud. If we assume that the total pressure (kinetic plus magnetic) is constant from 1810 to 1830 , the proton perpendicular temperature is estimated to be on the order of 70 eV.

In the magnetospheric tail, the resonances are weaker and shorter than in the inner magnetosphere. They are also more closely spaced in frequency (typically $1 \mathrm{kHz}$ or less). Even in this case the magnetospheric version of the program of automatic determination of the plasma frequency gives satisfactory results. When the gyrofrequency is too low to be resolved by the experiment, an estimate of the plasma frequency is given by the frequency of maximum intensity of the smoothed spectrum. This method has been used by Etcheto and Saint-Marc [1985] to study the plasma sheet boundary layer.

\section{SUMmary AND CONCLUSIONS}

The relaxation sounder, even though it has been widely used in passive mode to study waves spontaneously generated by the plasma [Christiansen et al., 1978a, b; Etcheto et al., 1982; Etcheto and Faucheux, 1984; Lacombe et al., 1985], is primarily an active experiment, aimed at exciting radio waves at the characteristic frequencies of the medium, thus bringing information on the local plasma. The most interesting of this information is a reliable and accurate determination of the electron density. In order to make the instrument easily usable for routinely measuring the electron density it was necessary
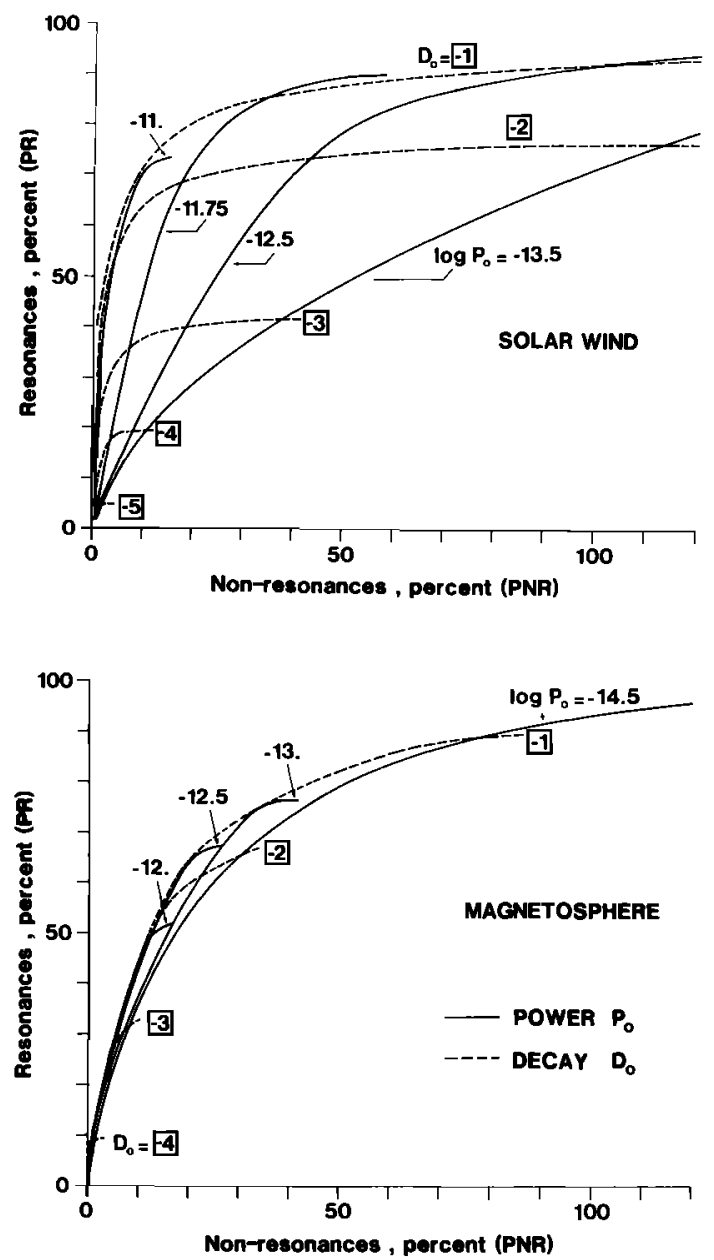

Fig. 15. Percentages of resonances (ordinate) and nonresonances (abscissa) having a power larger than $P_{0}$ and a decay smaller than $D_{0}$ in the solar wind (top) and magnetosphere (bottom). While in the solar wind a simple threshold on the "power" and "decay" enables us to roughly sort out most of the resonances, this is no longer true in the magnetosphere.

to develop an automatic method for determining the plasma frequency, working in any region visited by the satellite. We realized this tool for the relaxation sounder flown on board ISEE 1.

In the solar wind and magnetosheath, where only one strong and long-lasting resonance is observed close to the plasma Irequency, we used a signal pattern recognition method, the quadratic discrimination of Sebestyen [1962]. Each recognized resonance has a quality coefficient enabling the user to sort out the results according to the use he wants to make of them. A proper sorting enables us to recognize more than $95 \%$ of the resonances, thus determining the density with an accuracy of $4 \%$ for a $20-\mathrm{kHz}$ plasma frequency.

In the magnetosphere where many resonances are observed, such a method is no longer usable since the signal pattern between various resonances is more different than it is between a resonance and a nonresonance. We therefore compare the observations to the theoretical dispersion relation of the Bernstein's modes. The electron gyrofrequency is determined with an accuracy better than $0.7 \%$. The plasma frequency of the coldest population is determined with an accuracy better than $10 \%$ in $93 \%$ of the cases while the total plasma frequency is given within $10 \%$. A direct check of these results cannot be made since no experiment is able to give the total 


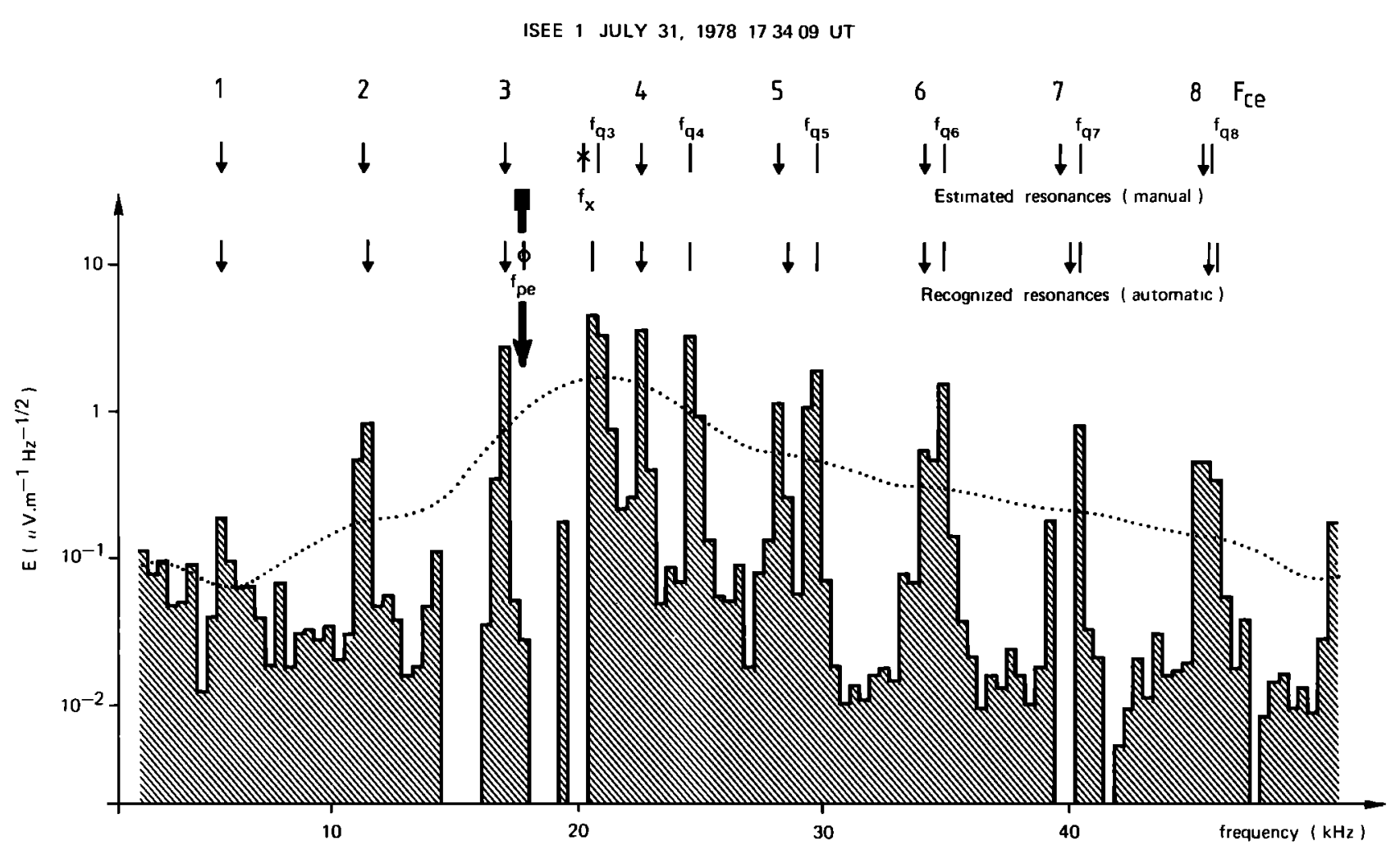

Fig. 16. Power spectrum in the magnetosphere (hatched curve) and smoothed spectrum (dotted line). Results of manual identification of resonances from analog telemetry (top arrows) and automatic identification from digital telemetry (series below). The maximum of the spectrum is slightly above the plasma frequency. The agreement between manual and automatic identification is good.

plasma frequency nor the electron distribution in the magnetosphere with such an accuracy [Decreau et al., 1978; Burke and Young, 1981].

The relaxation sounder, just like the natural wave experiments, is not subject to disturbances coming from the floating potential of the spacecraft. The density determinations relying on natural waves use several methods: thermal noise of the plasma (in the solar wind), low-frequency cutoff of the nonthermal continuum radiation (when it is local), line at the so-called "upper hybrid frequency" (when it is present and unambiguously identified). None of these methods can be systematically used since the natural noise is not always present. Moreover no automatic data reduction is possible since the natural noise has first to be identified, which is not necessarily straightforward. Even by visual inspection of the data a misinterpretation is easily possible when there is no cross-check with an active experiment [Jones, 1982; Etcheto et al., 1985]. In addition, one should be aware that resonances are much stronger than natural waves, usually by about $60 \mathrm{~dB}$, and therefore do not require very sensitive receivers. At last, if resonances correspond to clearly defined local characteristic frequencies of the medium (vanishing group velocity), it is not the same for natural waves. Indeed, either they are seen at instable frequencies which depend on the unknown free energy of the particle distribution ("upper hybrid noise") or they show a cutoff which is not necessarily local (nonthermal continuum cutoff corresponds to the maximum plasma frequency along the path followed by the wave between the source and the observer).

A relaxation sounder, coupled with an appropriate plasma frequency recognition program, is a very powerful tool for diagnosing the local plasma in the solar-terrestrial environ- ment and should become part of the payloads flown in this region, as a standard experiment in the same way as magnetometers.

\section{APPENDIX: DEFINITION OF THE VARIABLES USED IN the Automatic Recognition Algorithm FOR SOLAR WIND RESONANCES}

The signal (the electric field) is known through $N$ samples made on a frequency step: $\left\{\left(E_{n}, t_{n}\right)\right.$ with $\left.n=1, \cdots, N\right\}$ where $E_{n}$ is the $n$th measurement made on the step at time $t_{n}$ from the beginning of the step, after removing the noise level of the instrument. The signal received at the beginning of the step, before the AGC has reached its maximum value (19 $\mathrm{ms}$ for the 2-ms time constant of the AGC), is discarded.

We first constitute by manual means a reference set of $M$ resonances without any frequency-discriminating process. This is done by taking into account the knowledge we have of a resonance pattern. Let us now assume that a resonance is described by

$$
\ln E=A \ln t+B
$$

where $A$ and $B$ are respectively the slope and the value of $\ln E$ at $t=1$ for one resonance. $A$ and $B$ are given by

$$
\begin{gathered}
A=\left[\sum_{n=1}^{N}\left(\ln t_{n}-\langle\ln t\rangle\right)\left(\ln E_{n}-\langle\ln E\rangle\right)\right] \\
\cdot\left[\sum_{n=1}^{N}\left(\ln t_{n}-\langle\ln t\rangle\right)^{2}\right]^{-1} \\
B=\langle\ln E\rangle-A\langle\ln t\rangle
\end{gathered}
$$

where 


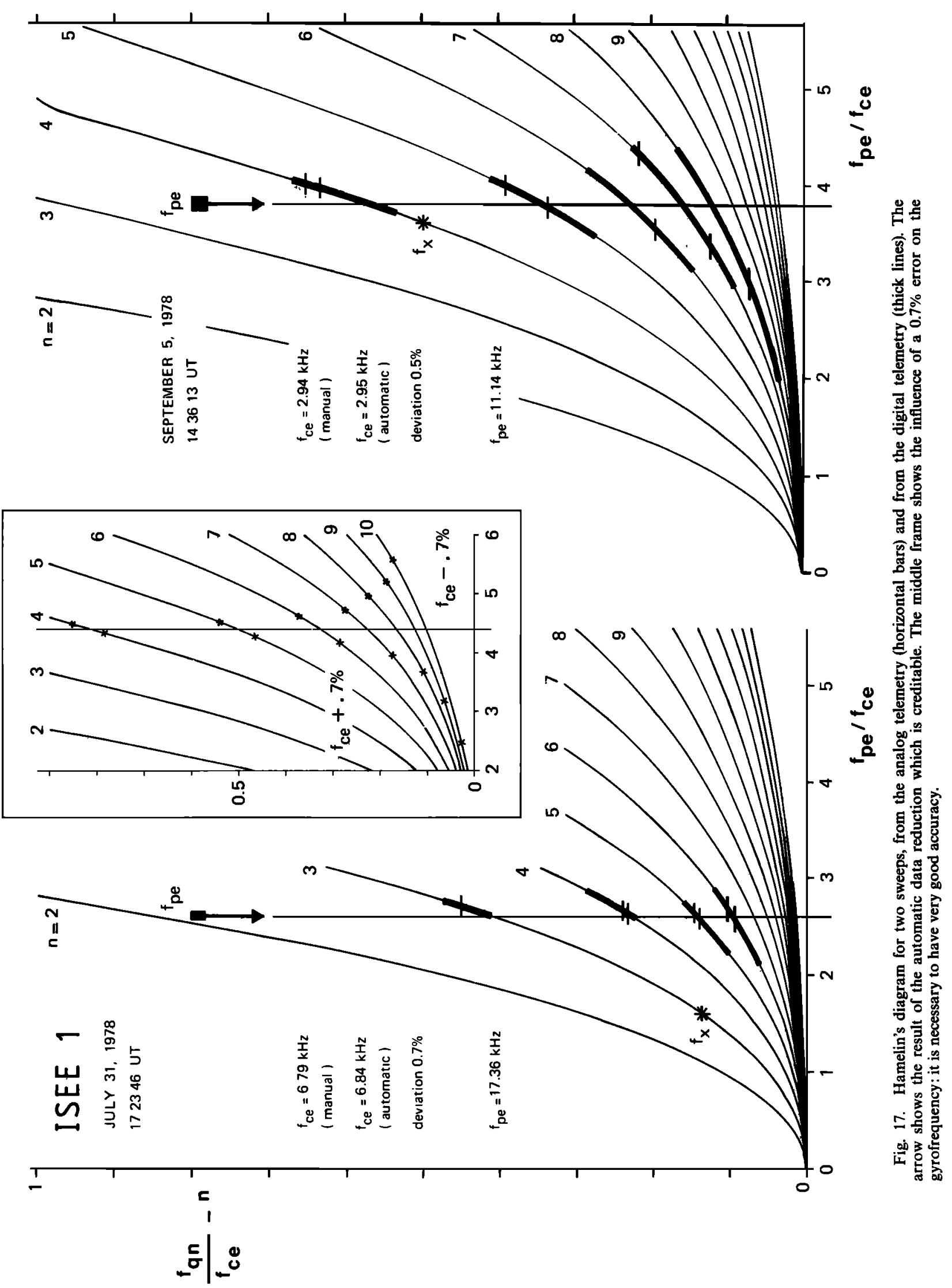




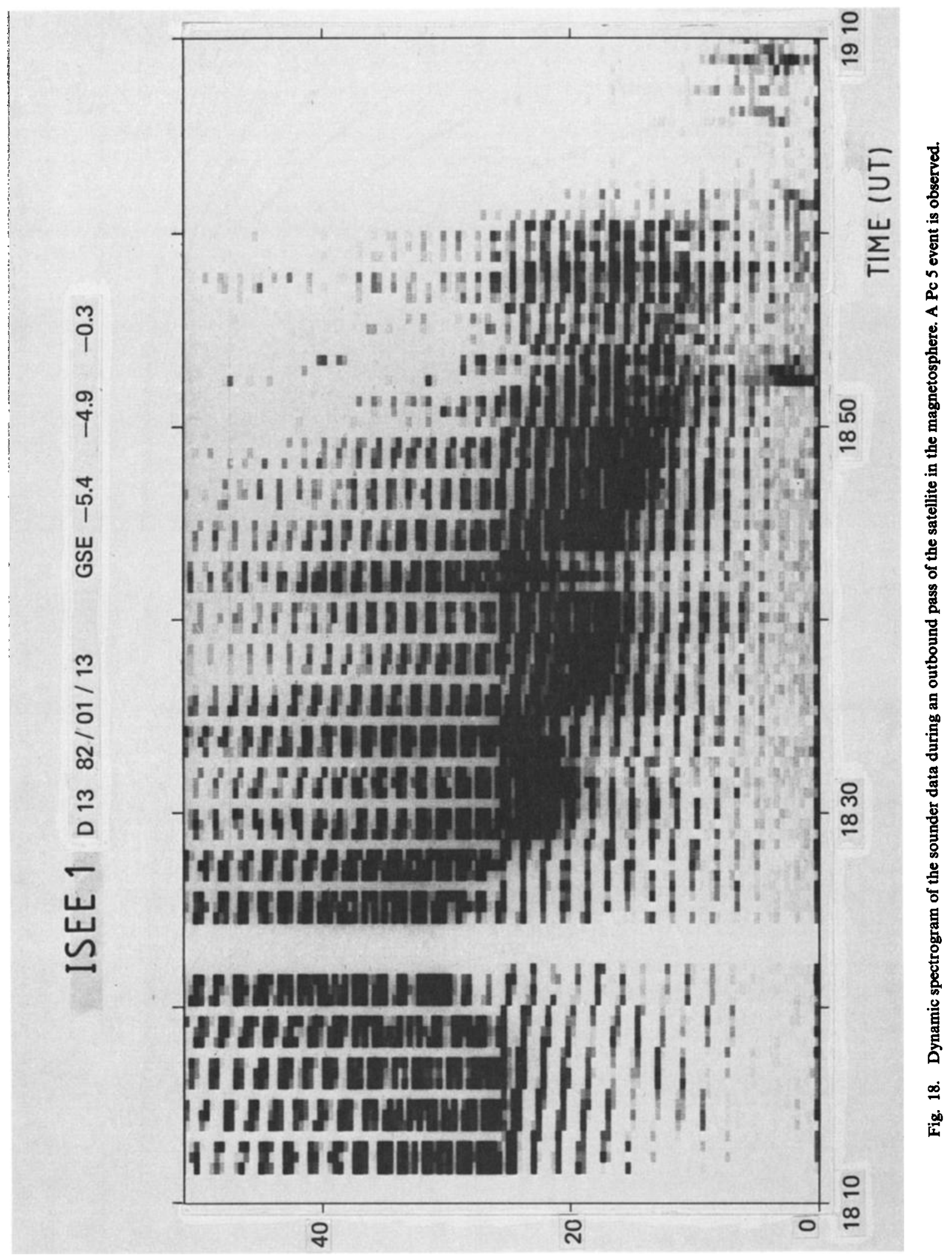


ISEE 1 JANUARY 13, 1982

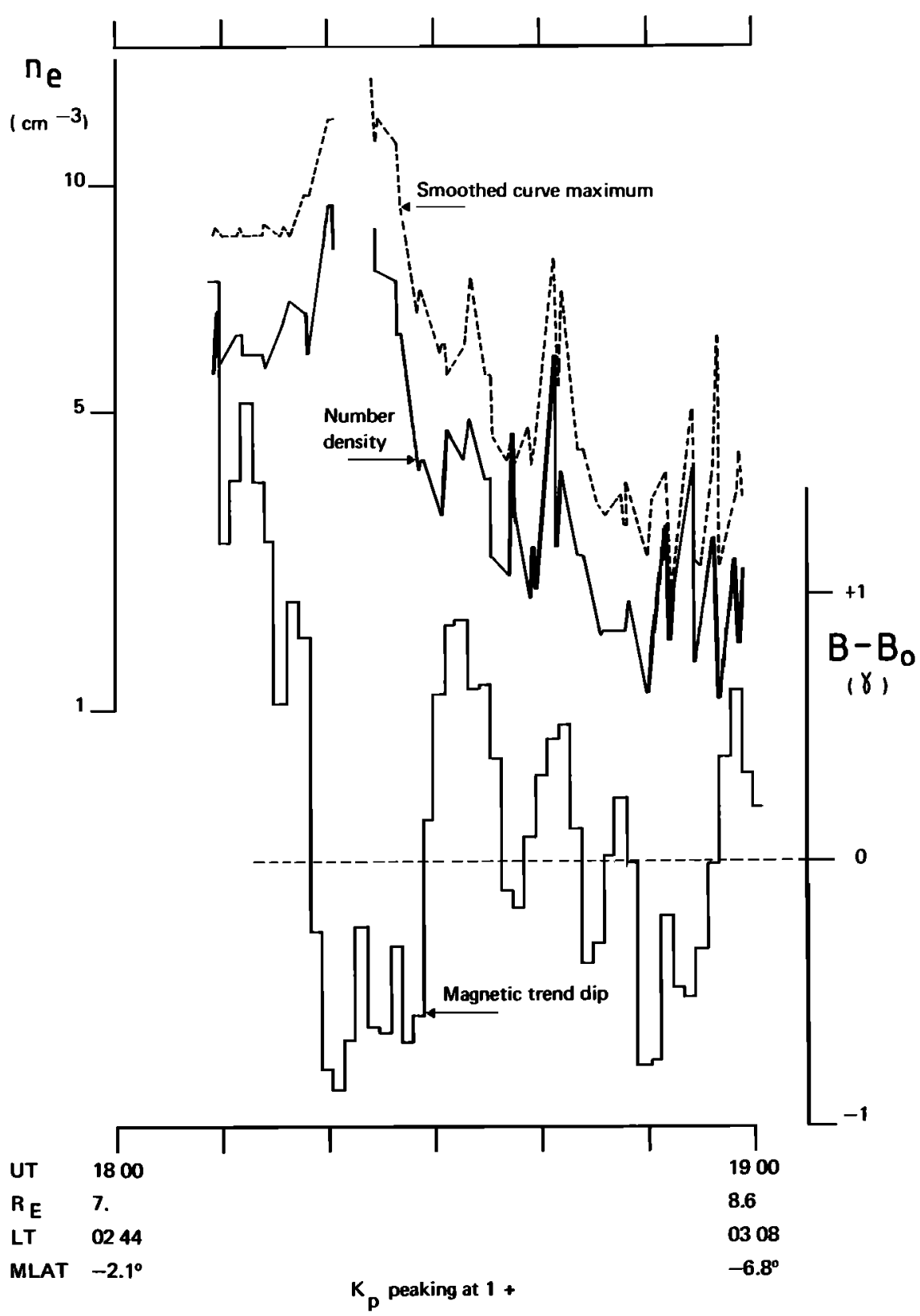

Fig. 19. Results of the automatic data reduction during the same period as in Figure 18. At the top: density corresponding to the frequency of the maximum of the smoothed spectrum (dashed line) and to the $f_{q m}$ alignment (solid line). At the bottom: detrended magnetic field from the magnetometer. The density and magnetic field fluctuations of the Pc 5 are in phase. A plasma cloud inducing a diamagnetic effect is seen between 1815 and 1830.

$$
\langle\ln t\rangle=\frac{1}{N} \sum_{n=1}^{N} \ln t_{n} \quad\langle\ln E\rangle=\frac{1}{N} \sum_{n=1}^{N} \ln E_{m}
$$

Let $\langle A\rangle$ and $\langle B\rangle$ be the average values of $A$ and $B$ calculated for all of the $M$ resonances of the reference set; the "average resonance" is then defined as

$$
\ln E=\langle A\rangle \ln t+\langle B\rangle
$$

We have now to define the three variables which best describe a resonance. These variables serve as inputs in the automatic recognition algorithm we applied in the solar wind data.
Let $P$ be the average, on a frequency step, of the electric field weighted by the "average resonance" defined previously:

$$
P=\frac{1}{N} \sum_{n=1}^{N} E_{n} \exp \left(\langle A\rangle \ln t_{n}+\langle B\rangle\right)
$$

Let us now consider all the frequency steps of a given frequency sweep. We can compute $\langle P\rangle$, the average value of $P$ for this sweep. Then the variable VAR1, improperly called "power," will be

$$
\text { VARI }=P /\langle P\rangle
$$


for each frequency step which belongs to the sweep under study. The second variable, VAR2, called "decay," is merely the slope of the signal in the frequency step.

$$
\mathrm{VAR} 2=\boldsymbol{A}
$$

Notice that VAR2 is an exponent ( $E$ is varying as $t^{A}$ ) and is consequently dimensionless.

Finally the third variable, VAR3, called "deviation," is the deviation of the signal measured on a frequency step from the signal called "average resonance" above.

$$
\text { VAR3 }=\frac{1}{N} \sum_{n=1}^{N}\left(\frac{\langle A\rangle \ln t_{n}+\langle B\rangle-\ln E_{n}}{\langle A\rangle \ln t_{n}+\langle B\rangle}\right)^{2}
$$

Acknowledgments. C. C. Harvey has played an important part in making the experiment successful: the authors wish to thank him here. This work was supported by Centre National d'Etudes Spatiales under contract 214.

The Editor thanks D. B. Muldrew for his assistance in evaluating this paper.

\section{REFERENCES}

Belmont, G., Characteristic frequencies of a non-Maxwellian plasma: A method for localizing the exact frequencies of magnetospheric intense natural waves near $f_{\text {pe }}$ Planet. Space Sci., 29, 1251, 1981.

Benson, R. F., Stimulated plasma waves in the ionosphere, Radio Sci., 12, 861, 1977.

Bernstein, I. B., Waves in a plasma in a magnetic field, Phys. Rev., $109,10,1958$.

Burke, W. J., and D. T. Young, Magnetic fields, low energy plasma, and wave measurements in the radiation belts, in Proceedings of the Air Force-Geophysics Laboratory Workshop on the Earth's Radiation Belts, Rep. AFGL-TR-8I-0311, pp. 209-243, Air Force Geophys. Lab., Bedford, Mass., 1981.

Calvert, W., Resonances in the ionosphere, in Plasma Waves in Space and Laboratory, edited by J. O. Thomas and B. J. Landmark, pp. 41-54, Edinburgh University Press, 1969.

Christiansen, P. J., M. P. Gough, G. Martelli, J. J. Bloch, N. Cornilleau-Wehrlin, J. Etcheto, R. Gendrin, D. Jones, C. Beghin, and $P$. Decreau, GEOS 1: Identification of natural magnetospheric emissions, Nature, 272, 682, 1978 .

Christiansen, P. J., M. P. Gough, G. Martelli, J. J. Bloch, N. Cornilleau-Wehrlin, J. Etcheto, R. Gendrin, C. Beghin, P. Decreau, and D. Jones, GEOS 1: Observations of electrostatic waves and their relationships with plasma parameters, Space Sci. Rev., 4, 383, $1978 b$.

Decreau, P. M. E., J. Etcheto, K. Knott, A. Pedersen, G. L. Wrenn, and $D$. T. Young, Multi-experiment determination of plasma density and temperature, Space Sci. Rev., 22, 633, 1978.

Deering, W. D., and J. A. Fejer, Excitation of plasma resonances by a small pulsed dipole, Phys. Fluids, 8, 2066, 1965.

De Feraudy, H., and M. Hamelin, Analysis and theoretical approach of the $f_{q}$ magnetospheric plasma resonances excited by the GEOS spacecraft relaxation sounder, paper presented at XIXth General Assembly, Commission H Symposium, URSI, Helsinki, 1978.

Etcheto, J., and J. J. Bloch, Plasma density measurements from the GEOS-1 relaxation sounder, Space Sci. Rev., 22, 597, 1978.

Etcheto, J., and $M$. Faucheux, Detailed study of electron plasma waves upstream of the earth's bow shock, J. Geophys. Res., 89, $6631,1984$.

Etcheto, J., and M. Petit, Une nouvelle méthode de caractérisation du plasma magnétosphérique, C. R. Acad. Sci., 285, 329, 1977.

Etcheto, J., and A. Saint-Marc, Anomalously high plasma densities in the plasma sheet boundary layer, J. Geophys. Res., 90, 5338, 1985.

Etcheto, J., H. de Feraudy, and J. G. Trotignon, Plasma resonance simulation in space plasmas, in Active Experiments in Space Plasmas, edited by C. T. Russell and M. J. Rycroft, p. 183, Pergamon, New York, 1981.
Etcheto, J., P. J. Christiansen, M: P: Gough, and J. G. Trotignon, Terrestrial continuum radiation observations with GEOS-1 and ISEE-1, Geophys. Res. Lett., 9, 1239, 1982.

Etcheto, J., G. Belmont, P. Canu, and J. G. Trotignon, Active sounder experiments on GEOS and ISEE, in Active Experiments in Space, Eur. Space Agency Spec. Publ., ESA-SP 95, 39, 1983:

Etcheto, J., P. J. Christiansen, and M. P. Gough, Problems in the interpretation of natural plasma wave spectra, Ann. Geophys. Gauthier Villars, 3, 417, 1985.

Fejer, J. A., and W.-M. Yu, Excitation of plasima resonances by a small pulsed dipole in a weakly inhomogeneous plasma, J. Geophys. Res., 75, 1919, 1970.

Franklin, C. A., and M. A. Maclean, The design of swept-frequency topside sounders, Special Issue on Topside Sounding and the Ionosphere, Proc. IEEE, 57, 897, 1969.

Hamelin, M., Propagation and difection radiation properties of the cyclotron harmonic waves in a maxwelliah plasma, paper prèsented at International Conference on Plasma Physics, Int. Union of Pure and Appl. Phys., URSI, Nagoya, Japan, 1980.

Harvey, C. C., J. Etcheto, Y. de Javel, R. Mánning, and M. Petit, The ISEE electron experiment, IEEE Trans. Geosci. Electron., GE-16, 231, 1978.

Harvey, C. C., J. Etcheto, and A. Mangeney, Early results from the ISEE electron density experiment, Space Sci. Rev., 23, 39, 1979.

Jones, D., Terrestrial myriametric radiation from the earth's plasmapause, Planet. Space Sci., 30, 399, 1982.

Kivelson, M. G., J. Etcheto, and J. G. Trotignon, Global compressional oscillations of the terrestrial magnetosphere: The evidence and a model, J. Geophys. Res., 89, $9851,1984$.

Lacombe, C., A. Mangeney, C: C. Harvey, and J. D. Scudder, Electron plasma waves upstream of the earth's bow shock, J. Geophys. Res., 90, 73, 1985.

McAfee, J. R., Topside resonances as oblique echoes, J. Geophys. Res., 74, 802, 1969.

McAfee, J. R., Electron plasma resonances in the topside ionosphere, Fundam. Cosmic Phys., I, 71, 1973.

Muldrew, D. B., Electrostatic resonances associated with the maximum frequencies of cyclotron-harmonic waves, J. Geophys. Res., 77, 1794, 1972a.

Muldrew, D. B., Electron resonances observed with topside sounders, Radio Sci., 7, 779, $1972 b$.

Pottelette, R., M. Hamelin, J. M. Illiano, and B. Lembège, Interpretation of the fine structure of electrostatic waves excited in space, Phys. Fluids, 24, 1517, 1981.

Romeder, J. M., Méthodes et Programmes d'Analyse Discriminante, Dunod, Paris, 1973.

Sebestyen, G. S., Decision-Making Processes in Pattern Recognition, Macmillan, New York, 1962.

Singer, H. J., C. T. Russell, M. G. Kivelson, T. A. Fritz, and W. Lennartsson, Satellite observations of the spatial extent and structure of $\mathrm{Pc} 3,4,5$ pulsations near the magnetospheric equator, Geophys. Res. Lett., 6, 889, 1979.

Thouvenin, J. P., and J. G. Trotignon, Automatic resonance recognition method in solar wind, Nuovo Ciment. Soc. Ital. Fis. C, 3, 696, 1980.

Trotignon, J. G., J. Etcheto, and J. P. Thouvenin, Automatic determination of the electron density measured by the relaxation sounder onboard ISEE 1 satellite (abstract), Eos Trans. AGU, 63, 1320, 1982.

J. Etcheto, Centre de Recherche en Physique de l'Environnement Terrestre et Planétaire, 38-40 rue du Général Leclerc, 92131 Issy les Moulineaux, France.

J. P. Thouvenin, Centre National d'Etudes Spatiales, 18 Avenue Edouard Belin, 31055 Toulouse Cedex, France.

J. G. Trotignon, Laboratoire de Physique et Chimie de l'Environnement, 3 A Avenue de la Recherche Scientifique, 45071 Orléans Cedex 2, France.

(Received April 5, 1985; revised July 12,1985 ; accepted July 24,1985 .) 